\title{
The abelianization of the level 2 mapping class group
}

\author{
Masatoshi Sato
}

\begin{abstract}
In this paper, we determine the abelianization of the level $d$ mapping class group for $d=2$ and odd $d$. We also extend the homomorphism of the Torelli group defined by Heap to a homomorphism of the level 2 mapping class group.
\end{abstract}

\section{Contents}

1 Introduction 2

2 The abelianization of the level $d$ congruence subgroup of symplectic group 4

2.1 Proof of Proposition 2.1 . . . . . . . . . . . . . . . . . . . . . . . 5

3 The abelianization of the level 2 mapping class group 8

3.1 Spin structures of mapping tori . . . . . . . . . . . . . . . . . . . . . . . . 8

3.2 A spin manifold bounded by Mapping tori . . . . . . . . . . . . . . . . . . . . . . 9

3.3 The homomorphism $\beta_{\sigma, x}$ on the level 2 mapping class group . . . . . . . . . . . . . . . . 10

3.4 Brown invariant . . . . . . . . . . . . . . . . . . . . . . . . 11

3.5 Heap's homomorphism . . . . . . . . . . . . . . . . . . . . . . . . 12

3.6 The value of $\beta_{\sigma, x} \ldots \ldots \ldots \ldots \ldots$

4 Proof of Theorem $1.2 \quad 16$

4.1 A homomorphism $\Phi: \mathbf{Z}\left[S_{d}\right] \rightarrow H_{1}\left(\mathcal{M}_{g, r}[d] ; \mathbf{Z}\right) \ldots \ldots \ldots$

4.2 Upper bound of the order $\left|H_{1}\left(\mathcal{M}_{g, 1}[2] ; \mathbf{Z}\right)\right| \ldots \ldots \ldots$. . . . . . . . . . . . . . 17

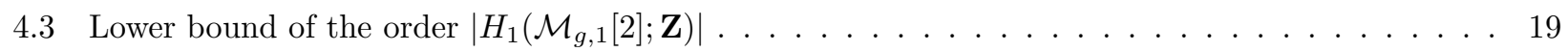

4.4 The abelianization of the level 2 mapping class group of a closed surface . . . . . . . . . 22

5 The abelianization of the level $d$ mapping class group for odd $d \quad 23$

5.1 Mod $d$ reduction of inclusion homomorphism . . . . . . . . . . . . . . . . . . 23

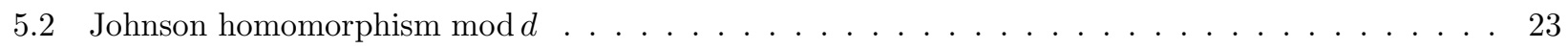




\section{Introduction}

Let $g \geq 1, r=0,1$, and $d>0$. We denote by $\Sigma_{g, r}$ a closed oriented connected surface of genus $g$ with $r$ boundary components. We denote by Diff $+\left(\Sigma_{g, r}, \partial \Sigma_{g, r}\right)$ the group of orientation-preserving diffeomorphisms of $\Sigma_{g, r}$ which fix the boundary pointwise. The mapping class group of $\Sigma_{g, r}$ is defined by $\mathcal{M}_{g, r}:=\pi_{0} \operatorname{Diff}_{+}\left(\Sigma_{g, r}, \partial \Sigma_{g, r}\right)$. Fix the symplectic basis $\left\{A_{i}, B_{i}\right\}_{i=1}^{g}$ of the first homology group $H_{1}\left(\Sigma_{g, r} ; \mathbf{Z}\right)$. Then the natural action of $\mathcal{M}_{g, r}$ on this group gives rise to the classical representation $\rho: \mathcal{M}_{g, r} \rightarrow \operatorname{Sp}(2 g ; \mathbf{Z})$ onto the integral symplectic group. The kernel $\mathcal{I}_{g, r}$ of this representation is called the Torelli group.

The level $d$ mapping class group $\mathcal{M}_{g, r}[d] \subset \mathcal{M}_{g, r}$ is defined by the kernel of the mod $d$ reduction $\mathcal{M}_{g, r} \rightarrow$ $\operatorname{Sp}\left(2 g ; \mathbf{Z}_{d}\right)$ of $\rho$. The level $d$ congruence subgroup $\Gamma_{g}[d]$ of the symplectic group is defined by the kernel of $\bmod d$ reduction map $\operatorname{Ker}\left(\operatorname{Sp}(2 g ; \mathbf{Z}) \rightarrow \operatorname{Sp}\left(2 g ; \mathbf{Z}_{d}\right)\right)$. This is equal to the image of $\mathcal{M}_{g, r}[d]$ under $\rho$. The group $\mathcal{M}_{g}[d]$ arises as the orbifold fundamental group of the moduli space of nonsingular curves of genus $g$ with level $d$ structure. In particular, for $d \geq 3$, the level $d$ mapping class groups are torsion-free, and the abelianizations of the level $d$ mapping class groups are equal to the first homology groups of the corresponding moduli spaces.

In this paper, we determine the abelianization of this group $\mathcal{M}_{g, r}[d]$ and $\Gamma_{g}[d]$, for $d=2$ and odd $d$ when $g \geq 3$. This is an analogous result in Satoh[22] and Lee-Szczarba[17] for the abelianizations of the level $d$ congruence subgroups of Aut $F_{n}$ and $\operatorname{GL}(n ; \mathbf{Z})$. To determine the abelianization $H_{1}\left(\mathcal{M}_{g, r}[2] ; \mathbf{Z}\right)$, we construct an injective homomorphism $\beta_{\sigma}: \mathcal{M}_{g, 1}[2] \rightarrow \operatorname{Map}\left(H_{1}\left(\Sigma_{g} ; \mathbf{Z}_{2}\right), \mathbf{Z}_{8}\right)$. This function is defined using the Rochlin functions of mapping tori. We will show that this is an extension of a homomorphism of the Torelli group defined by Heap [6]. To determine the abelianization $H_{1}\left(\mathcal{M}_{g, r}[d] ; \mathbf{Z}\right)$ for odd $d$, we construct the Johnson homomorphism of modulo $d$ on $\mathcal{M}_{g, r}[d]$.

Historically, McCarthy 18 proved that the first rational homology group of a finite index subgroup of $\mathcal{M}_{g, r}$ which includes the Torelli group vanishes for $r=0$. More generally, Hain [5] proved that this group vanishes for any $r \geq 0$.

Theorem 1.1 (McCarthy, Hain). Let $\mathcal{M}$ be a finite index subgroup of $\mathcal{M}_{g, r}$ that includes the Torelli group, where $g \geq 3, r \geq 0$. Then

$$
H_{1}(\mathcal{M} ; \mathbf{Q})=0 .
$$

Farb raised the problem to compute the abelianization, that is the first integral homology group, of the group $\mathcal{M}_{g, r}[d]$ in Farb 4] Problem 5.23 p.43. Recently, Putman[20] also determined the abelianization of the level $d$ congruence subgroup of the symplectic group and the level $d$ mapping class group for odd $d$ when $g \geq 3$. See also [21.

This paper is organized as follows. In section 2, we determine the commutator subgroup of the level $d$ congruence subgroup of the symplectic group for every integer $d \geq 2$. This is mainly relies on the work of Mennicke [19] and Bass-Milnor-Serre [1] on congruence subgroups of the symplectic group. We also obtain the abelianization of $\Gamma_{g}[d]$ (Corollary 2.2). Let $\operatorname{spin}(M)$ be the set of spin structures of an oriented manifold $M$ with trivial second Stiefel-Whitney class. In section 3, we will construct the injective homomorphism $\beta_{\sigma}: \mathcal{M}_{g, 1}[2] \rightarrow$ $\operatorname{Map}\left(H_{1}\left(\Sigma_{g} ; \mathbf{Z}_{2}\right), \mathbf{Z}_{8}\right)$ for $\sigma \in \operatorname{spin}\left(\Sigma_{g}\right)$. We will determine the abelianization of the level 2 mapping class group using this homomorphism. Let $n$ be a positive integer. Denote the Rochlin function by $R(M):, \operatorname{spin}(M) \rightarrow \mathbf{Z}_{16}$ for a $4 n-1$-manifold $M$. For $\sigma \in \operatorname{spin}(M), R(M, \sigma)$ is defined as the signature of a compact $4 n$-manifold which spin bounds $(M, \sigma)$. See for example Turaev[23. We will define the homomorphism $\beta_{\sigma}(\varphi)$ using the difference $R(M, \sigma)-R\left(M, \sigma^{\prime}\right)$ for a mapping torus $M=M_{\varphi}$ of $\varphi \in \mathcal{M}_{g, 1}[2]$. Turaev[23] proved that it can be written as the pin $^{-}$bordism class of a surface embedded in the mapping torus. We can compute $\beta_{\sigma}$ by examining this pin $^{-}$bordism class.

The main theorem in this paper proved in Section 4 is illustrated as follows. For $\left\{x_{i}\right\}_{i=1}^{n} \subset H_{1}\left(\Sigma_{g, 1} ; \mathbf{Z}_{2}\right)$, 
define $I: H_{1}\left(\Sigma_{g, 1} ; \mathbf{Z}_{2}\right)^{n} \rightarrow \mathbf{Z}_{2}$ by

$$
I\left(x_{1}, x_{2}, \cdots, x_{n}\right):=\sum_{1 \leq i<j \leq n}\left(x_{i} \cdot x_{j}\right) \bmod 2,
$$

where $x_{i} \cdot x_{j}$ is the intersection number of $x_{i}$ with $x_{j}$. We denote by $\mathbf{Z}_{8}\left[H_{1}\left(\Sigma_{g, 1} ; \mathbf{Z}_{2}\right)\right]$ the free $\mathbf{Z}_{8}$-module generated by all formal symbol $[X]$ for $X \in H_{1}\left(\Sigma_{g, 1}\right)$. Define $\Delta_{0}^{n}: H_{1}\left(\Sigma_{g, 1} ; \mathbf{Z}\right)^{n} \rightarrow \mathbf{Z}_{8}\left[H_{1}\left(\Sigma_{g, 1} ; \mathbf{Z}_{2}\right)\right]$ by

$$
\begin{array}{r}
\Delta_{0}^{n}\left(x_{1}, x_{2}, \cdots, x_{n}\right)=\sum_{i=1}^{n}\left[x_{i}\right]+\sum_{1 \leq i<j \leq n}(-1)^{I\left(x_{i}, x_{j}\right)}\left[x_{i}+x_{j}\right]+\sum_{1 \leq i<j<k \leq n}(-1)^{I\left(x_{i}, x_{j}, x_{k}\right)}\left[x_{i}+x_{j}+x_{k}\right] \\
+\cdots+(-1)^{I\left(x_{1}, x_{2}, \cdots, x_{n}\right)}\left[x_{1}+x_{2}+x_{3}+\cdots+x_{n}\right] \in \mathbf{Z}_{8}\left[H_{1}\left(\Sigma_{g, 1} ; \mathbf{Z}_{2}\right)\right] .
\end{array}
$$

Theorem 1.2. Let $g \geq 3$. Denote by $L_{g, 1} \subset \mathbf{Z}_{8}\left[H_{1}\left(\Sigma_{g, 1} ; \mathbf{Z}_{2}\right)\right]$ the submodule generated by

$$
[0], 4 \Delta_{0}^{2}\left(x_{1}, x_{2}\right), 2 \Delta_{0}^{3}\left(x_{1}, x_{2}, x_{3}\right), \Delta_{0}^{n}\left(x_{1}, x_{2}, \cdots, x_{n}\right) \in \mathbf{Z}_{8}\left[H_{1}\left(\Sigma_{g, 1} ; \mathbf{Z}_{2}\right)\right]
$$

for $n \geq 3$ and $\left\{x_{i}\right\}_{i=1}^{n} \subset H_{1}\left(\Sigma_{g, 1} ; \mathbf{Z}_{2}\right)$. Then, we have

$$
\mathbf{Z}_{8}\left[H_{1}\left(\Sigma_{g, 1} ; \mathbf{Z}_{2}\right)\right] / L_{g, 1} \cong H_{1}\left(\mathcal{M}_{g, 1}[2] ; \mathbf{Z}\right)
$$

as an $\mathcal{M}_{g, 1}$-module.

We also determine the abelianization of the level 2 mapping class group of a closed surface in subsection 4.4 . In section 5, we determine the abelianization of the level $d$ mapping class group for odd $d$. The main tool is the Johnson homomorphism of modulo $d$ on the level $d$ mapping class group. This derives from the extension of the Johnson homomorphism defined by Kawazumi[14. For $H:=H_{1}\left(\Sigma_{g, r} ; \mathbf{Z}\right)$, denote by $\Lambda^{3} H / H$ the cokernel of the homomophism

$$
\begin{array}{ccc}
H & \rightarrow & \Lambda^{3} H \\
x & \mapsto & \sum_{i=1}^{g}\left(A_{i} \wedge B_{i}\right) \wedge x .
\end{array}
$$

Then, the abelianization of the level $d$ mapping class group is written as:

Theorem 1.3. For $g \geq 3$ and odd integer $d \geq 3$,

$$
\begin{aligned}
H_{1}\left(\mathcal{M}_{g}[d] ; \mathbf{Z}\right) & =\left(\Lambda^{3} H / H \otimes \mathbf{Z}_{d}\right) \oplus H_{1}\left(\Gamma_{g}[d] ; \mathbf{Z}\right) \\
& =\mathbf{Z}_{d}^{\left(4 g^{3}-g\right) / 3} \\
H_{1}\left(\mathcal{M}_{g, 1}[d] ; \mathbf{Z}\right) & =\left(\Lambda^{3} H \otimes \mathbf{Z}_{d}\right) \oplus H_{1}\left(\Gamma_{g}[d] ; \mathbf{Z}\right) \\
& =\mathbf{Z}_{d}^{\left(4 g^{3}+5 g\right) / 3} .
\end{aligned}
$$




\section{The abelianization of the level $d$ congruence subgroup of symplec- tic group}

In this section, we determine the abelianization of the level $d$ congruence subgroup $\Gamma_{g}[d]$ of the symplectic group $\operatorname{Sp}(2 g ; \mathbf{Z})$. We denote the identity matrix by $I$. A matrix $A \in \Gamma_{g}[d]$ can be written as $A=I+d A^{\prime}$ with an integral $2 g \times 2 g$ matrix $A^{\prime}$. Denote the matrix

$$
A^{\prime}=\left(\begin{array}{ll}
p(A) & q(A) \\
r(A) & s(A)
\end{array}\right)
$$

where $p(A), q(A), r(A)$, and $s(A)$ are $g \times g$ matrices. We also denote the $(i, j)$-element of a matrix $u$ by $u_{i j}$. For an even integer $d$, define the subgroup $\Gamma_{g}[d, 2 d]$ of the symplectic group by

$$
\Gamma_{g}[d, 2 d]:=\left\{A \in \Gamma_{g}[d] \mid q_{i i}(A)=r_{i i}(A) \equiv 0 \bmod 2 \text { for } i=1,2 \cdots, g\right\} .
$$

This subgroup was proved to be the normal subgroup of $\operatorname{Sp}(2 g ; \mathbf{Z})$ in Igusa [8] Lemma 1.(i).

We will prove in this section:

Proposition 2.1. Let $g \geq 2$. For an odd integer $d$,

$$
\Gamma_{g}\left[d^{2}\right]=\left[\Gamma_{g}[d], \Gamma_{g}[d]\right]
$$

For an even integer $d$,

$$
\Gamma_{g}\left[d^{2}, 2 d^{2}\right]=\left[\Gamma_{g}[d], \Gamma_{g}[d]\right]
$$

Before proving Proposition 2.1, we calculate the abelianization of the congruence subgroup $\Gamma_{g}[d]$ using this proposition. First, we compute the module $\Gamma_{g}[d] / \Gamma_{g}\left[d^{2}\right] \cong H_{1}\left(\Gamma_{g}[d] ; \mathbf{Z}\right)$ when $d$ is an odd integer. For $A:=I+d A^{\prime}, B=I+d B^{\prime} \in \Gamma_{g}[d]$, we have

$$
A B=I+d\left(A^{\prime}+B^{\prime}\right) \bmod d^{2} .
$$

Hence, we can define the surjective homomorphism $m: \Gamma_{g}[d] \rightarrow \mathbf{Z}_{d}^{2 g^{2}+g}$ by

$$
m(A):=\left(\left\{p_{i j}(A)\right\}_{1 \leq i \leq g, 1 \leq j \leq g},\left\{q_{i j}(A)\right\}_{1 \leq i \leq j \leq g},\left\{r_{i j}(A)\right\}_{1 \leq i \leq j \leq g}\right) \bmod d .
$$

This is the restriction of the homomorphism of the level $d$ congruence subgroup of $\operatorname{SL}(2 g ; \mathbf{Z})$ defined by Lee and Szczarba 17. From the symplectic condition, we obtain $p(A)+{ }^{t} s(A) \equiv 0, q(A) \equiv{ }^{t} q(A)$, and $r(A) \equiv{ }^{t} r(A)$ $\bmod d$. Then, we have the exact sequence

$$
1 \longrightarrow \Gamma_{g}\left[d^{2}\right] \longrightarrow \Gamma_{g}[d] \stackrel{m}{\longrightarrow} \mathbf{Z}_{d}^{2 g^{2}+g} \longrightarrow 1 .
$$

This shows that $H_{1}\left(\Gamma_{g}[d] ; \mathbf{Z}\right) \cong \mathbf{Z}_{d}^{2 g^{2}+g}$.

Next, we consider the case when $d$ is even. We compute the group $\Gamma_{g}[d] / \Gamma_{g}\left[d^{2}, 2 d^{2}\right] \cong H_{1}\left(\Gamma_{g}[d] ; \mathbf{Z}\right)$. By the exact sequence (2), we have another exact sequence

$$
0 \longrightarrow \frac{\Gamma_{g}\left[d^{2}\right]}{\Gamma_{g}\left[d^{2}, 2 d^{2}\right]} \longrightarrow \frac{\Gamma_{g}[d]}{\Gamma_{g}\left[d^{2}, 2 d^{2}\right]} \stackrel{m}{\longrightarrow} \mathbf{Z}_{d}^{2 g^{2}+g} \longrightarrow 0
$$

For a matrix

$$
A=I+d^{2}\left(\begin{array}{ll}
p(A) & q(A) \\
r(A) & s(A)
\end{array}\right) \in \Gamma_{g}\left[d^{2}\right]
$$


define the surjective homomorphism $m_{1}^{\prime}: \Gamma_{g}\left[d^{2}\right] \rightarrow \mathbf{Z}_{2}^{2 g}$ by

$$
m_{1}^{\prime}(A):=\left(\left\{q_{i i}(A)\right\}_{i=1}^{g},\left\{r_{i i}(A)\right\}_{i=1}^{g}\right) \bmod 2 .
$$

The kernel is equal to $\Gamma_{g}\left[d^{2}, 2 d^{2}\right]$. Hence, this induces the isomorphism $\Gamma_{g}\left[d^{2}\right] / \Gamma_{g}\left[d^{2}, 2 d^{2}\right] \cong \mathbf{Z}_{2}^{2 g}$. The exact sequence (3) is consequently wrriten as

$$
0 \longrightarrow \mathbf{Z}_{2}^{2 g} \longrightarrow \frac{\Gamma_{g}[d]}{\Gamma_{g}\left[d^{2}, 2 d^{2}\right]} \stackrel{m}{\longrightarrow} \mathbf{Z}_{d}^{2 g^{2}+g} \longrightarrow 0
$$

For a homology class $y \in H_{1}\left(\Sigma_{g, r} ; \mathbf{Z}\right)$, define the transvection $T_{y} \in \operatorname{Sp}(2 g ; \mathbf{Z})$ by $T_{y}(x):=x+(y \cdot x) y$. Then by the exact sequence (4), we see that Ker $m$ is generated by the elements $T_{A_{i}}^{d^{2}}, T_{B_{i}}^{d^{2}}$, where $i=1,2, \cdots, g$. Since $q_{i i}\left(T_{A_{i}}^{d}\right)=1$ and $r_{i i}\left(T_{B_{i}}^{d}\right)=1$, the order of $T_{A_{i}}^{d}, T_{B_{i}}^{d} \in \Gamma_{g}[d] / \Gamma_{g}\left[d^{2}, 2 d^{2}\right]$ are $2 d$. Hence we have:

Corollary 2.2. For $g \geq 2$,

$$
H_{1}\left(\Gamma_{g}[d] ; \mathbf{Z}\right)= \begin{cases}\mathbf{Z}_{d}^{2 g^{2}+g} & \text { if d is odd } \\ \mathbf{Z}_{d}^{2 g^{2}-g} \oplus \mathbf{Z}_{2 d}^{2 g} & \text { if d is even }\end{cases}
$$

\subsection{Proof of Proposition 2.1}

In this subsection, we prove Proposition 2.1

By the equation (11), we have

$$
\left[\Gamma_{g}[d], \Gamma_{g}[d]\right] \subset \Gamma_{g}\left[d^{2}\right]
$$

for every $d \geq 2$. In particular, if $d$ is even, it is shown that

$$
\left[\Gamma_{g}[d], \Gamma_{g}[d]\right] \subset \Gamma_{g}\left[d^{2}, 2 d^{2}\right]
$$

in Igusa [8] Lemma 1.(ii). Hence, it suffices to prove

$$
\begin{aligned}
& \Gamma_{g}\left[d^{2}\right] \subset\left[\Gamma_{g}[d], \Gamma_{g}[d]\right], \text { for } d \text { odd, and } \\
& \Gamma_{g}\left[d^{2}, 2 d^{2}\right] \subset\left[\Gamma_{g}[d], \Gamma_{g}[d]\right], \text { for } d \text { even. }
\end{aligned}
$$

First, we show that $2 d\left[T_{A_{1}}^{d}\right]=0$ for every $d$. A straightforward computation shows the following lemma.

Lemma 2.3. For $g \geq 2$ and $d \geq 1$,

$$
T_{a_{1} A_{1}+b_{1} B_{1}+a_{2} A_{2}}^{d}=\left(T_{A_{2}}^{d}\right)^{\left(a_{1} b_{1}+1\right) a_{2}^{2}}\left(T_{B_{1}+A_{2}}^{d} T_{A_{2}}^{-d} t_{B_{1}}^{-d}\right)^{b_{1} a_{2}}\left(T_{A_{1}+A_{2}}^{d} t_{A_{1}}^{-d} T_{A_{2}}^{-d}\right)^{a_{1} a_{2}} T_{a_{1} A_{1}+b_{1} B_{1}}^{d} .
$$

If we put $a_{1}=1, a_{2}=-1, b_{1}=0$, we obtain

$$
\left[T_{A_{1}+A_{2}}^{d}\right]+\left[T_{A_{1}-A_{2}}^{d}\right]=2\left[T_{A_{1}}^{d}\right]+2\left[T_{A_{2}}^{d}\right] .
$$

Let $x, y \in H_{1}\left(\Sigma_{g, r} ; \mathbf{Z}\right)$ be elements such that $x \cdot y=0$ and $\{x, y\}$ can be extended to form a basis of $\left.H_{1}\left(\Sigma_{g, r} ; \mathbf{Z}\right)\right)$. Then, there exists $\varphi \in \mathcal{M}_{g, r}$ which satisfies $\varphi_{*}(x)=A_{1}$, and $\varphi_{*}(y)=A_{2}$. This shows that:

Lemma 2.4. If $x, y \in H_{1}\left(\Sigma_{g, r} ; \mathbf{Z}\right)$ satisfy $x \cdot y=0$, and $\{x, y\}$ can be extended to form a basis of $H_{1}\left(\Sigma_{g, r} ; \mathbf{Z}\right)$, then we have

$$
\left[T_{x+y}^{d}\right]+\left[T_{x-y}^{d}\right]=2\left[T_{x}^{d}\right]+2\left[T_{y}^{d}\right]
$$


Remark 2.5. For $i=1,2,3,4$, let $D_{i} \subset S^{2}$ be mutually disjoint disks. By the assumption of $x, y$ in Lemma 2.4. we can choose an embedding $i: S^{2}-\amalg_{i=1}^{4} D_{i} \rightarrow \Sigma_{g, r}$ such that $\left[i\left(\partial D_{1}\right)\right]=-\left[i\left(\partial D_{2}\right)\right]=x$, and $\left[i\left(\partial D_{3}\right)\right]=$ $-\left[i\left(\partial D_{4}\right)\right]=y$. The Lantern relation of this embedding

$$
T_{x+y} T_{x-y}=T_{x}^{2} T_{y}^{2} \in \mathrm{Sp}(2 g ; \mathbf{Z})
$$

also shows the above relation.

Put $x=k A_{1}+A_{2}, y=A_{1}$ in the equation of Lemma 2.4, and take the summation over $k=1,2, \cdots, d-1$. Then, we have

$$
2 d\left[T_{A_{1}}^{d}\right]=0 \in H_{1}\left(\Gamma_{g}[d] ; \mathbf{Z}\right) .
$$

Next, we show that $d\left[T_{A_{1}}^{d}\right]=0$ when $d$ is odd. By the equation (5) and Lemma 2.4, we have

$$
d\left[T_{x+2 k y}^{d}\right]=d\left[T_{x+2(k+1) y}^{d}\right]
$$

for $k \in \mathbf{Z}$. If $d$ is odd, we obtain

$$
d\left[T_{x}^{d}\right]=d\left[T_{x+y}^{d}\right]=d\left[T_{y}^{d}\right] .
$$

If we put $a_{1}=b_{1}=2$, and $a_{2}=1$ in Lemma 2.3, we have

$$
\left[T_{2 A_{1}+2 B_{1}+A_{2}}^{d}\right]=5\left[T_{A_{2}}\right]^{d}+2\left(\left[T_{B_{1}+A_{2}}^{d}\right]-\left[T_{A_{2}}^{d}\right]-\left[T_{B_{1}}^{d}\right]\right)+2\left(\left[T_{A_{1}+A_{2}}^{d}\right]-\left[T_{A_{1}}^{d}\right]-\left[T_{A_{2}}^{d}\right]\right)+\left[T_{2 A_{1}+2 B_{1}}^{d}\right] .
$$

If we apply the equation (6) to $d$ times the equation (7), we have

$$
d\left[T_{A_{1}}^{d}\right]=0 \in H_{1}\left(\Gamma_{g}[d] ; \mathbf{Z}\right) .
$$

We need the theorem proved by Mennicke[19], which is essential in this proof.

Theorem 2.6 (Mennicke). Let $g \geq 2$ and $d>0$. If $Q$ is a normal subgroup of $\operatorname{Sp}(2 g ; \mathbf{Z})$ which contains $T_{A_{1}}^{d}$, then

$$
\Gamma_{g}[d] \subset Q .
$$

By the equation (8), we have $T_{A_{1}}^{d^{2}} \in\left[\Gamma_{g}[d], \Gamma_{g}[d]\right]$ when $d$ is odd. By the equation (5), we also have $T_{A_{1}}^{2 d^{2}} \in\left[\Gamma_{g}[d], \Gamma_{g}[d]\right]$ when $d$ is even. Hence we obtain

$$
\begin{gathered}
\Gamma_{g}\left[2 d^{2}\right] \subset\left[\Gamma_{g}[d], \Gamma_{g}[d]\right] \text { if } d \text { is even, and } \\
\Gamma_{g}\left[d^{2}\right] \subset\left[\Gamma_{g}[d], \Gamma_{g}[d]\right] \text { if } d \text { is odd. }
\end{gathered}
$$

Thus, we have proved the case when $d$ is odd. To prove the case when $d$ is even, it suffices to show:

Lemma 2.7. If $d$ is even,

$$
\Gamma_{g}\left[d^{2}, 2 d^{2}\right] \subset\left[\Gamma_{g}[d], \Gamma_{g}[d]\right] .
$$

Proof. We have already known that $\Gamma_{g}\left[2 d^{2}\right] \subset\left[\Gamma_{g}[d], \Gamma_{g}[d]\right]$. Hence, we examine the quotient group $\Gamma_{g}\left[d^{2}, 2 d^{2}\right] / \Gamma_{g}\left[2 d^{2}\right]$. The symplectic group $\operatorname{Sp}(2 g ; \mathbf{Z})$ acts on $\Gamma_{g}\left[d^{2}, 2 d^{2}\right] / \Gamma_{g}\left[2 d^{2}\right]$ by the conjugation action. For $1 \leq i, j \leq 2 g$, denote the $2 g \times 2 g$ matrix $e_{i j}$ which has 1 in the $(i, j)$-element, and 0 in the other elements. First, we prove that $\Gamma_{g}\left[d^{2}, 2 d^{2}\right] / \Gamma_{g}\left[2 d^{2}\right]$ is generated by $I+d^{2}\left(e_{1 g+2}+e_{2 g+1}\right), I+d^{2}\left(e_{11}-e_{g+1 g+1}\right)$ as a $\operatorname{Sp}(2 g ; \mathbf{Z})$-module.

Similar to the homomorphism $m$, for a matrix

$$
A=I+d^{2}\left(\begin{array}{ll}
p(A) & q(A) \\
r(A) & s(A)
\end{array}\right) \in \Gamma_{g}\left[d^{2}, 2 d^{2}\right]
$$


we define the surjective homomorphism $m_{2}^{\prime}: \Gamma_{g}\left[d^{2}, 2 d^{2}\right] \rightarrow \mathbf{Z}_{2}^{2 g^{2}-g}$ by

$$
m_{2}^{\prime}:=\left(\left\{p_{i j}(A)\right\}_{1 \leq i \leq g, 1 \leq j \leq g},\left\{q_{i j}(A)\right\}_{1 \leq i<j \leq g},\left\{r_{i j}(A)\right\}_{1 \leq i<j \leq g}\right) \bmod 2
$$

Then, it is easy to see that $\operatorname{Ker} m_{2}^{\prime}=\Gamma_{g}\left[2 d^{2}\right]$, and $m_{2}^{\prime}$ induces the isomorphism $\Gamma_{g}\left[d^{2}, 2 d^{2}\right] / \Gamma_{g}\left[2 d^{2}\right] \cong \mathbf{Z}_{2}^{2 g^{2}-g}$. For $i, j$ such that $1 \leq i, j \leq g, i \neq j$, there are elements of $\operatorname{Sp}(2 g$; Z $)$ which map 4-tuple of homology classes $\left(A_{1}, A_{2}, B_{1}, B_{2}\right)$ to

$$
\left(A_{i}, A_{j}, B_{i}, B_{j}\right),\left(B_{i}, B_{j},-A_{i},-A_{j}\right), \text { and }\left(A_{i},-B_{j}, B_{i}, A_{j}\right),
$$

respectively. By the conjugation action, these elements send $I+d^{2}\left(e_{1 g+2}+e_{2 g+1}\right)$ to

$$
I+d^{2}\left(e_{i g+j}+e_{j g+i}\right), I+d^{2}\left(e_{g+i j}+e_{g+j i}\right), \text { and } I+d^{2}\left(e_{i j}-e_{g+j g+i}\right) \in \Gamma_{g}\left[d^{2}, 2 d^{2}\right] / \Gamma_{g}\left[2 d^{2}\right]
$$

respectively. Denote the Kronecker delta by $\delta_{i j}$. Then we have

$$
p_{k l}\left(I+d^{2}\left(e_{i g+j}+e_{j g+i}\right)\right)=q_{k l}\left(I+d^{2}\left(e_{g+i j}+e_{g+j i}\right)\right)=r_{k l}\left(I+d^{2}\left(e_{i j}-e_{g+j g+i}\right)\right)=\delta_{i k} \delta_{j l} .
$$

In the same way, there is an element of $\operatorname{Sp}(2 g ; \mathbf{Z})$ which map the pair $\left(A_{1}, B_{1}\right)$ to $\left(A_{i}, B_{i}\right)$. This element sends $I+d^{2}\left(e_{11}-e_{g+1 g+1}\right)$ to $I+d^{2}\left(e_{i i}-e_{g+i g+i}\right)$. Note that

$$
p_{k k}\left(I+d^{2}\left(e_{i i}+e_{g+i g+i}\right)\right)=\delta_{i k}
$$

Then we see that from the equations (9) and (10), $\Gamma_{g}\left[d^{2}, 2 d^{2}\right] / \Gamma_{g}\left[2 d^{2}\right] \cong \mathbf{Z}_{2}^{2 g^{2}-g}$ is generated by the elements $I+d^{2}\left(e_{1 g+2}+e_{2 g+1}\right), I+d^{2}\left(e_{11}-e_{g+1 g+1}\right)$ as a $\operatorname{Sp}(2 g ; \mathbf{Z})$-module.

Next, we will show that

$$
I+d^{2}\left(e_{11}-e_{g+1 g+1}\right), I+d^{2}\left(e_{1 g+2}+e_{2 g+1}\right) \in\left[\Gamma_{g}[d], \Gamma_{g}[d]\right]
$$

For $A=I+d A^{\prime}, B=I+d B^{\prime} \in \Gamma_{g}[d]$, we have

$$
A B A^{-1} B^{-1} \equiv I+d^{2}\left(A^{\prime} B^{\prime}-B^{\prime} A^{\prime}\right) \bmod d^{3} .
$$

If we put $A^{\prime}=e_{1 g+1}, B^{\prime}=e_{g+11}$, and $A^{\prime}=e_{12}-e_{g+2 g+1}, B^{\prime}=e_{2 g+2}$, we get (11).

The fact (11) shows

$$
\Gamma_{g}\left[d^{2}, 2 d^{2}\right] \subset\left[\Gamma_{g}[d], \Gamma_{g}[d]\right]
$$

This proves the lemma.

Hence, we complete the proof of Proposition 2.1 . 


\section{The abelianization of the level 2 mapping class group}

In this section, we will define a family of homomorphisms

$$
\beta_{\sigma, x}: \mathcal{M}_{g, 1}[2] \rightarrow \Omega_{2}^{p i n^{-}} \cong \mathbf{Z}_{8}
$$

for $\sigma \in \operatorname{spin}\left(\Sigma_{g}\right)$ and $x \in H_{1}\left(\Sigma_{g} ; \mathbf{Z}_{2}\right)$ (Lemma 3.2). This family determines the abelianization of the level 2 mapping class group. The homomorphism $\beta_{\sigma, x}$ is proved to be an extension of the homomorphism $\omega_{\sigma, y}$ defined by Heap [6] to the level 2 mapping class group (Subsection [3.5). We will calculate the values of this homomorphism on generators of the level 2 mapping class group using the Brown invariant (Proposition 3.8).

\subsection{Spin structures of mapping tori}

Fix a closed disk neighborhood $N\left(c_{0}\right)$ of a point $c_{0}$ in $\Sigma_{g}$. The mapping class group $\pi_{0} \operatorname{Diff}_{+}\left(\Sigma_{g}, N\left(c_{0}\right)\right)$ is the group of isotopy classes of orientation-preserving diffeomorphisms of $\Sigma_{g}$ which fix the neighborhood $N\left(c_{0}\right)$ pointwise. By restricting each diffeomorphism to $\Sigma_{g}-\operatorname{Int} N\left(c_{0}\right)$, the group $\pi_{0} \operatorname{Diff}_{+}\left(\Sigma_{g}, N\left(c_{0}\right)\right)$ is isomorphic to $\mathcal{M}_{g, 1}$. Hence, we identify these two groups. We also identify the kernel $\operatorname{Ker}\left(\pi_{0} \operatorname{Diff}+\left(\Sigma_{g}, N\left(c_{0}\right)\right) \rightarrow \operatorname{Sp}\left(2 g ; \mathbf{Z}_{2}\right)\right)$ of the mod 2 reduction of $\rho$ with $\mathcal{M}_{g, 1}[2]$.

For $\varphi=[f] \in \mathcal{M}_{g, 1}$, denote the mapping torus of $\varphi$ by $M:=M_{\varphi}:=\Sigma_{g} \times[0,1] / \sim$, where the equivalence relation is given by $(f(x), 0) \sim(x, 1)$. In this subsection, we define a map $\theta: \operatorname{spin}\left(\Sigma_{g}\right) \rightarrow \operatorname{spin}\left(M_{\varphi}\right)$ for $\varphi \in \mathcal{M}_{g, 1}[2]$.

First, we define the spin structure of an oriented vector bundle. Let $E \rightarrow V$ be a smooth oriented vector bundle of rank $n$ on a smooth manifold $V$. We denote by $P(E)$ the oriented frame bundle associated to this bundle. When the Stiefel-Whitney class $w_{2}$ of $E$ vanishes, we define the spin structure of $E$ by a right inverse homomorphism of the natural homomorphism $H_{1}\left(P(E) ; \mathbf{Z}_{2}\right) \rightarrow H_{1}\left(V ; \mathbf{Z}_{2}\right)$. Denote by $\operatorname{spin}(E)$ the set of spin structure of $E$. Since $P(E)$ is a principal $G L_{+}(n)$ bundle and $w_{2}$ vanishes, the Serre spectral sequence shows that

$$
0 \longrightarrow H^{1}\left(V ; \mathbf{Z}_{2}\right) \longrightarrow H^{1}\left(P(E) ; \mathbf{Z}_{2}\right) \longrightarrow \mathbf{Z}_{2} \longrightarrow 0
$$

is exact. Define the injective map

$$
\operatorname{spin}(E) \rightarrow H^{1}\left(P(E) ; \mathbf{Z}_{2}\right)
$$

by $\sigma \mapsto v$, where $v$ is the unique nontrivial element in $\operatorname{Ker} \sigma$. The element $v \in H^{1}\left(P(E)\right.$; $\left.\mathbf{Z}_{2}\right)$ restricts to an non-trivial element in each fiber of $P(E) \rightarrow V$. This is also equivalent to consider the double cover of the orthonormal frame bundle associated to the bundle $E$ with a fiber metric. In detail, for example, see Lee-MillerWeintraub[16] Section 1.1. For an oriented smooth $n$-manifold $V$, we define the spin structure of $V$ by the spin structure of the tangent bundle $T V$. We denote $\operatorname{simply}$ by $\operatorname{spin}(V):=\operatorname{spin}(T V)$ the set of spin structure on $V$. Note that a spin structure of $V$ is equivalent to a spin structure of $V \times(-\epsilon, \epsilon)^{k}$, for $\epsilon>0$ and $k>0$.

Next, we define the injective map $\theta: \operatorname{spin}\left(\Sigma_{g}\right) \rightarrow \operatorname{spin}\left(M_{\varphi}\right)$. Fix a spin structure on $\Sigma_{g}$. Since $\varphi \in \mathcal{M}_{g, 1}[2]$ acts on $H_{1}\left(\Sigma_{g} ; \mathbf{Z}_{2}\right)$ trivially, the Wang exact sequence is written as

$$
0 \longrightarrow H_{1}\left(\Sigma_{g} ; \mathbf{Z}_{2}\right) \longrightarrow H_{1}\left(M ; \mathbf{Z}_{2}\right) \longrightarrow H_{1}\left(S^{1} ; \mathbf{Z}_{2}\right) \longrightarrow 0 .
$$

The inclusion map $l: N\left(c_{0}\right) \times S^{1} \rightarrow M$ gives the splitting

$$
H_{1}\left(M ; \mathbf{Z}_{2}\right)=H_{1}\left(\Sigma_{g} ; \mathbf{Z}_{2}\right) \oplus H_{1}\left(S^{1} ; \mathbf{Z}_{2}\right) \text {. }
$$

In order to define the spin structure on $M$, we will construct homomorphisms from each direct summand to $H_{1}\left(P(M) ; \mathbf{Z}_{2}\right)$. For $N\left(c_{0}\right) \times S^{1} \subset M$, define the framing $\hat{l}: S^{1} \rightarrow P\left(N\left(c_{0}\right) \times S^{1}\right)$ by $\hat{l}(t)=\left(v_{0} \cos 2 \pi t+\right.$ 
$\left.v_{1} \sin 2 \pi t, v_{1} \cos 2 \pi t-v_{0} \sin 2 \pi t, v_{S^{1}}(t)\right)$, where $\left\{v_{0}, v_{1}\right\}$ is a frame of $T_{c_{0}} N\left(c_{0}\right)$, and $v_{S^{1}}(t) \in T_{t} S^{1}$ is a nonzero tangent vector. This framing induces the homomorphism

$$
H_{1}\left(S^{1} ; \mathbf{Z}_{2}\right) \stackrel{\hat{\imath}}{\longrightarrow} H_{1}\left(P\left(N\left(c_{0}\right) \times S^{1}\right) ; \mathbf{Z}_{2}\right) \stackrel{\mathrm{inc}_{*}}{\longrightarrow} H_{1}\left(P(M) ; \mathbf{Z}_{2}\right),
$$

where inc $_{*}$ is the homomorphism induced by the inclusion map.

Next, consider the natural smooth map $P\left(\Sigma_{g} \times(-\epsilon, \epsilon)\right) \rightarrow P(M)$ induced by the inclusion of a tubular neighborhood $\Sigma_{g} \times(-\epsilon, \epsilon) \subset M$ for small $\epsilon$. Using the spin structure on $\Sigma_{g}$, we have the homomorphism

$$
H_{1}\left(\Sigma_{g} ; \mathbf{Z}_{2}\right) \stackrel{\sigma}{\longrightarrow} H_{1}\left(P\left(\Sigma_{g} \times(-\epsilon, \epsilon)\right) ; \mathbf{Z}_{2}\right) \stackrel{\text { inc }_{*}}{\longrightarrow} H_{1}\left(P(M) ; \mathbf{Z}_{2}\right) .
$$

Thus, we have constructed the homomorphism $H_{1}\left(M ; \mathbf{Z}_{2}\right) \rightarrow H_{1}\left(P(M) ; \mathbf{Z}_{2}\right)$. In this way, we obtain the map $\theta: \operatorname{spin}\left(\Sigma_{g}\right) \rightarrow \operatorname{spin}(M)$.

\subsection{A spin manifold bounded by Mapping tori}

Let $P_{0}:=S^{2}-\amalg_{i=1}^{3}$ Int $D_{i}$ denote a pair of pants, where $\left\{D_{i}\right\}$ are mutually disjoint disks and Int $D_{i}$ is interior of $D_{i}$ in $S^{2}$. Pick the paths $\alpha, \beta, \gamma \in \pi_{1}\left(P_{0}, x_{0}\right)$ going once round boundary components as in Figure 11. Denote by Diff $+\left(\Sigma_{g}, N\left(c_{0}\right)\right)[2]$ the kernel of the representation of $\operatorname{Diff}_{+}\left(\Sigma_{g}, N\left(c_{0}\right)\right)$ on $H_{1}\left(\Sigma_{g} ; \mathbf{Z}_{2}\right)$. Consider $\Sigma_{g}$ bundles with its structure group $\operatorname{Diff}_{+}\left(\Sigma_{g}, N\left(c_{0}\right)\right)[2]$. For $\varphi, \psi \in \mathcal{M}_{g, 1}[2]$, there exists a $\Sigma_{g}$ bundle $p: W=W_{\varphi, \psi} \rightarrow P_{0}$ such that the topological monodromy $\pi_{1}\left(P_{0}, x_{0}\right) \rightarrow \mathcal{M}_{g, 1}[2]$ sends $\alpha, \beta$, and $\gamma \in \pi_{1}\left(P_{0}, x_{0}\right)$ to $\varphi, \psi$, and $(\varphi \psi)^{-1} \in \mathcal{M}_{g, 1}[2]$, respectively. This bundle is unique up to diffeomorphism. Note that the boundary $\partial W$ is diffeomorphic to the disjoint sum $M_{\varphi} \amalg M_{\psi} \amalg M_{(\varphi \psi)^{-1}}$.

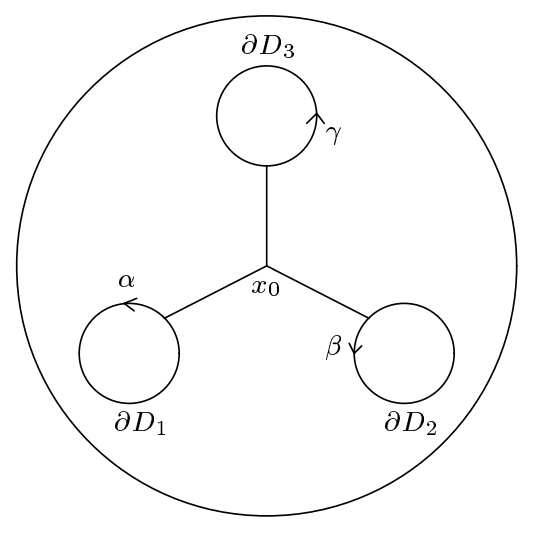

Figure 1: loops in a pair of pants

In this subsection, we define a spin structure of $W$. We show that the induced spin structure on each boundary component of $W$ is equal to that of the mapping torus defined in the last subsection.

Since $\varphi, \psi \in \mathcal{M}_{g, 1}[2]$ act on $H_{1}\left(\Sigma_{g} ; \mathbf{Z}_{2}\right)$ trivially, we have the splitting

$$
H_{1}\left(W ; \mathbf{Z}_{2}\right)=H_{1}\left(\Sigma_{g} ; \mathbf{Z}_{2}\right) \oplus H_{1}\left(P_{0} ; \mathbf{Z}_{2}\right)
$$

by the inclusion map $N\left(c_{0}\right) \times P_{0} \rightarrow W$. In order to define the spin structure on $W$, we will construct homomorphisms from each direct summand to $H_{1}\left(P(W) ; \mathbf{Z}_{2}\right)$. By the local triviality of the bundle $W \rightarrow P_{0}$, we have a neighborhood $\Sigma_{g} \times(-\epsilon, \epsilon)^{2} \subset W$ of the fiber on $x_{0} \in P_{0}$. Define the homomorphism

$$
H_{1}\left(\Sigma_{g} ; \mathbf{Z}_{2}\right) \stackrel{\sigma}{\longrightarrow} H_{1}\left(P\left(\Sigma_{g} \times(-\epsilon, \epsilon)^{2}\right) ; \mathbf{Z}_{2}\right) \stackrel{\text { inc }_{*}}{\longrightarrow} H_{1}\left(P(W) ; \mathbf{Z}_{2}\right) .
$$


Next, we will construct the homomorphism $H_{1}\left(P_{0} ; \mathbf{Z}_{2}\right) \rightarrow H_{1}\left(P(W) ; \mathbf{Z}_{2}\right)$. In the disk $D^{2}=\left\{(x, y) \in \mathbf{R}^{2} \mid x^{2}+\right.$ $\left.y^{2} \leq 1\right\}$, choose two mutually disjoint disks $D_{1}, D_{2} \subset \operatorname{Int} D^{2}$. Choose an orthonormal frame $\left\{v_{0}^{\prime}, v_{1}^{\prime}\right\}$ of $\mathbf{R}^{2}$. Let $s: D^{2}-D_{1}-D_{2} \rightarrow P\left(D^{2}-D_{1}-D_{2}\right)=\left(D^{2}-D_{1}-D_{2}\right) \times \mathbf{R}^{2}$ be the trivial framing defined by $s(x)=\left(x, v_{0}^{\prime}, v_{1}^{\prime}\right)$. By identifying $P_{0}$ with $D^{2}-D_{1}-D_{2}$, we have the map $\hat{l}^{\prime}: P_{0} \rightarrow P\left(N\left(c_{0}\right) \times P_{0}\right)$ by $\hat{l}^{\prime}(x)=\left(v_{0}, v_{1}, s(x)\right)$. This map and the inclusion $N\left(c_{0}\right) \times P_{0} \rightarrow W$ induce the homomorphism

$$
H_{1}\left(P_{0} ; \mathbf{Z}_{2}\right) \stackrel{\hat{l}^{\prime}}{\longrightarrow} H_{1}\left(P\left(N\left(c_{0}\right) \times P_{0}\right) ; \mathbf{Z}_{2}\right) \stackrel{\mathrm{inc}_{*}}{\longrightarrow} H_{1}\left(P(W) ; \mathbf{Z}_{2}\right) .
$$

Define the spin structure of $W$ by the homomorphisms (13) and (14).

Note that the homomorphism (13) is equal to the composite of (12) and the inclusion homomorphism $H_{1}\left(P(M \times[0, \epsilon)) ; \mathbf{Z}_{2}\right) \rightarrow H_{1}\left(P(W) ; \mathbf{Z}_{2}\right)$, where $M \times[0, \epsilon) \subset W$ is a collar neighborhood. We also see that the diagram

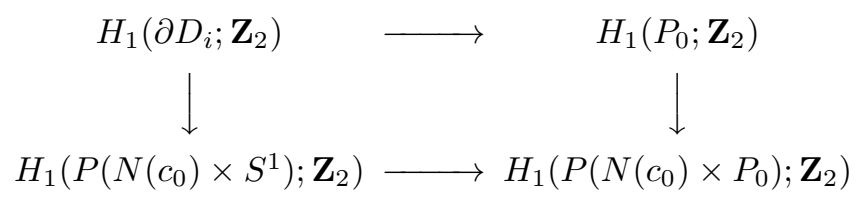

commutes. Hence, the manifold $W$ is spin bounded by $M_{\varphi}, M_{\psi}$, and $M_{(\varphi \psi)^{-1}}$ which were defined in the last subsection.

\subsection{The homomorphism $\beta_{\sigma, x}$ on the level 2 mapping class group}

In this subsection, we will construct a homomorphism which determines the abelianization of the group $\mathcal{M}_{g, 1}[2]$, using the Rochlin functions of mapping tori.

First we review the simply transitive action of $H_{1}\left(\Sigma_{g} ; \mathbf{Z}_{2}\right)$ on $\operatorname{spin}\left(\Sigma_{g}\right)$. Identify $H_{1}\left(\Sigma_{g} ; \mathbf{Z}_{2}\right)$ with $H^{1}\left(\Sigma_{g} ; \mathbf{Z}_{2}\right)$ by the Poincaré duality. By the Serre spectral sequence, we have the exact sequence

$$
1 \longrightarrow \mathbf{Z}_{2} \longrightarrow H_{1}\left(P\left(\Sigma_{g}\right) ; \mathbf{Z}_{2}\right) \longrightarrow H_{1}\left(\Sigma_{g} ; \mathbf{Z}_{2}\right) \longrightarrow 1
$$

For $x \in H^{1}\left(\Sigma_{g} ; \mathbf{Z}_{2}\right)=\operatorname{Hom}\left(H_{1}\left(\Sigma_{g} ; \mathbf{Z}_{2}\right), \mathbf{Z}_{2}\right)$, we denote again by $x: H_{1}\left(\Sigma_{g} ; \mathbf{Z}_{2}\right) \rightarrow H_{1}\left(P\left(\Sigma_{g}\right) ; \mathbf{Z}_{2}\right)$ the composite of $x: H_{1}\left(\Sigma_{g} ; \mathbf{Z}_{2}\right) \rightarrow \mathbf{Z}_{2}$ and the inclusion $\mathbf{Z}_{2} \subset H_{1}\left(P\left(\Sigma_{g}\right) ; \mathbf{Z}_{2}\right)$. Hence, for $\sigma \in \operatorname{spin}\left(\Sigma_{g}\right)$, we have another spin structure $\sigma+x: H_{1}\left(\Sigma_{g} ; \mathbf{Z}_{2}\right) \rightarrow H_{1}\left(P\left(\Sigma_{g}\right) ; \mathbf{Z}_{2}\right)$. In this way, $H_{1}\left(\Sigma_{g} ; \mathbf{Z}_{2}\right)$ acts on $\operatorname{spin}\left(\Sigma_{g}\right)$.

Every spin 3-manifold is known to bound a spin 4-manifold. For $\varphi \in \mathcal{M}_{g, 1}[2]$, choose a compact oriented spin manifold $V$ which is spin bounded by the mapping torus $M=M_{\varphi}$. Then the Rochlin function of $(M, \sigma)$ is defined by

$$
R(M, \sigma):=\operatorname{Sign} V \bmod 16 .
$$

This is well-defined by Rochlin's theorem, and is called the Rochlin function.

Definition 3.1. For $x \in H_{1}\left(\Sigma_{g} ; \mathbf{Z}_{2}\right)$ and $\sigma \in \operatorname{spin}\left(\Sigma_{g}\right)$, define the map

$$
\beta_{\sigma, x}: \mathcal{M}_{g, 1}[2] \rightarrow\left(\frac{1}{2} \mathbf{Z}\right) / 8 \mathbf{Z}
$$

by $\beta_{\sigma, x}(\varphi):=\left(R\left(M_{\varphi}, \theta(\sigma)\right)-R\left(M_{\varphi}\right), \theta(\sigma+x)\right) / 2 \bmod 8$.

Here, $\theta: \operatorname{spin}\left(\Sigma_{g}\right) \rightarrow \operatorname{spin}\left(M_{\varphi}\right)$ is the map defined in Subsection 3.2

Lemma 3.2. $\beta_{\sigma, x}$ is a homomorphism. 
Proof. As we saw in Subsection [3.2, for $\varphi, \psi \in \mathcal{M}_{g, 1}[2]$, the spin 4-manifold $W_{\varphi, \psi}$ is spin bounded by the mapping tori $M_{\varphi} \amalg M_{\psi} \amalg M_{(\varphi \psi)^{-1}}$. Therefore, we have

$$
\begin{gathered}
R\left(M_{\varphi}, \theta(\sigma)\right)+R\left(M_{\psi}, \theta(\sigma)\right)-R\left(M_{\varphi \psi}, \theta(\sigma)\right) \equiv \operatorname{Sign} W_{\varphi, \psi}, \\
R\left(M_{\varphi}, \theta(\sigma+x)\right)+R\left(M_{\psi}, \theta(\sigma+x)\right)-R\left(M_{\varphi \psi}, \theta(\sigma+x)\right) \equiv \operatorname{Sign} W_{\varphi, \psi} \bmod 16 .
\end{gathered}
$$

Hence, we have $\beta_{\sigma, x}(\varphi \psi)=\beta_{\sigma, x}(\varphi)+\beta_{\sigma, x}(\psi)$.

As we will show in Subsection 3.4, the image $\operatorname{Im} \beta_{\sigma, x}$ is in $\mathbf{Z}_{8}$. Denote by $\operatorname{Map}\left(H_{1}\left(\Sigma_{g} ; \mathbf{Z}_{2}\right) ; \mathbf{Z}_{8}\right)$ the free $\mathbf{Z}_{8}$-module consisting of all maps $H_{1}\left(\Sigma_{g} ; \mathbf{Z}_{2}\right) \rightarrow \mathbf{Z}_{8}$. We can define the homomorphism $\beta_{\sigma}: \mathcal{M}_{g, 1}[2] \rightarrow$ $\operatorname{Map}\left(H_{1}\left(\Sigma_{g} ; \mathbf{Z}_{2}\right) ; \mathbf{Z}_{8}\right)$ by $\beta_{\sigma}(\varphi)(x)=\beta_{\sigma, x}(\varphi)$.

\subsection{Brown invariant}

The $\operatorname{Pin}^{-}(n)$ group is a central extension of the $O(n)$ bundle. Let $F$ be a (not necessarily orientable) closed surface. A pin ${ }^{-}$structure of $F$ is defined by the principal $\mathrm{Pin}^{-}(2)$ bundle associated to the tangent bundle $T F$, where $\mathrm{Pin}^{-}(2)$ acts on $\mathbf{R}^{2}$ via its covering projection to $O(2)$. Brown defined the invariant of a closed surface $F$ with a pin $^{-}$structure, called the Brown invariant. In this subsection, We review this invariant and its relation to the Rochlin functions stated by Turaev 23 .

Definition 3.3. Let $F$ be a (not necessarily orientable) closed surface. If a function $\hat{q}: H_{1}\left(F ; \mathbf{Z}_{2}\right) \rightarrow \mathbf{Z}_{4}$ satisfies $\hat{q}(x+y)=\hat{q}(x)+\hat{q}(y)+2 x \cdot y$, we call $\hat{q}$ the quadratic enhancement.

On a closed surface $F$, a $\operatorname{pin}^{-}$structure $\alpha$ induces a quadratic enhancement $\hat{q}_{\alpha}$ as follows. Denote the determinant line bundle of the tangent bundle by $\operatorname{det} F$. Then $E:=T F \oplus \operatorname{det} F$ has a canonical orientation. The set of pin $^{-}$structures of $F$ is known to corresponds bijectively to the set of spin structures of $E$. In detail, see Kirby-Taylor [15]. For an element $v \in H_{1}\left(F ; \mathbf{Z}_{2}\right)$, choose a simple closed curve $K \subset F$ which represents $v$. Denote the normal bundle of $\left.T K \subset T F\right|_{K}$ and $\left.T F \subset E\right|_{F}$ by $N(F / K)$ and $N(E / F)$, respectively. Then the restriction $\left.E\right|_{K}$ can be written as $\left.E\right|_{K}=\left.T K \oplus N(F / K) \oplus N(E / F)\right|_{K}$. If we fix the orientation of $K$, the bundle $E^{\prime}=\left.N(F / K) \oplus N(E / F)\right|_{K}$ gets also oriented.

Choose a non-zero section $s_{K}: K \rightarrow T K$. We call a framing $s: K \rightarrow P\left(E^{\prime}\right)$ is odd if and only if the induced homomorphism $s \oplus s_{K}: H_{1}\left(K ; \mathbf{Z}_{2}\right) \rightarrow H_{1}\left(P(E) ; \mathbf{Z}_{2}\right)$ is not equal to the homomorphism $H_{1}\left(K ; \mathbf{Z}_{2}\right) \rightarrow$ $H_{1}\left(P(E) ; \mathbf{Z}_{2}\right)$ induced by the spin structure of $E$.

Definition 3.4. Choose an odd framing on K. Using it, count the number of right half twists that the odd framing of $K$ makes in a complete traverse with $F$. We denote this number by $\hat{q}_{\alpha}(v)$. This induces the map $\hat{q}_{\alpha}: H_{1}\left(F ; \mathbf{Z}_{2}\right) \rightarrow \mathbf{Z}_{4}$. We call it the quadratic enhancement of a pin ${ }^{-}$structure $\alpha$.

This number does not depend on the choice of the representative of a homology class, the orientation of $K$, and the odd framing of $N(F / K) \oplus N(E / F)$. In detail, see Kirby-Taylor [15] section 3. In particular, if $F$ is an orientable surface, this number is equal to twice the quadratic function induced by the spin structure on $F$.

Definition 3.5. Let $F$ be a closed surface with its pin ${ }^{-}$strucure $\alpha$. Then, the Brown invarant $B_{\alpha} \in \mathbf{Z}_{8}$ of $\alpha$ is defined by the equation

$$
\sqrt{\left|H_{1}\left(F ; \mathbf{Z}_{2}\right)\right|} \exp \left(2 \pi \sqrt{-1} B_{\alpha} / 8\right)=\sum_{x \in H_{1}\left(F ; \mathbf{Z}_{2}\right)} \exp \left(2 \pi \sqrt{-1} \hat{q}_{\alpha}(x) / 4\right) .
$$

Consider a closed surface $F$ which represents $s \in H_{2}\left(M ; \mathbf{Z}_{2}\right)$. Then, the surface $F$ has canonical pin ${ }^{-}$ structure induced by the spin structure of the tubular neighborhood of $F$. Furthermore, the pin ${ }^{-}$bordism class 
of $F$ does not depend on the representative of $s \in H_{2}\left(M ; \mathbf{Z}_{2}\right)$ (Kirby-Taylor [15] (4.8)). Denote the pin ${ }^{-}$bordism group by $\Omega_{*}^{p i n^{-}}$. It is known that the Brown invariant gives the isomorphism $\Omega_{2}^{\text {pin }} \cong \mathbf{Z}_{8}$ (Kirby-Taylor [15] Lemma 3.6). For $\sigma, \sigma^{\prime} \in \operatorname{spin}(M)$, Turaev 23] showed that the difference $R(M, \sigma)-R\left(M, \sigma^{\prime}\right)$ is written by the Brown invariant of the pin $^{-}$structure of an embedded surface.

Lemma 3.6 (Turaev [23] Lemma 2.3). Let $M$ be a closed manifold with its Stiefel-Whitney class $w_{2}=0$. Denote the closed surface $F \subset M$ which represents the Poincaré dual of $x \in H^{1}\left(M ; \mathbf{Z}_{2}\right)$. For a spin structure $\sigma$ of $M$, denote the induced pin ${ }^{-}$structure $\alpha$ of $F$. Then we have

$$
R(M, \sigma)-R(M, \sigma+x)=2 B_{\alpha} .
$$

Apply the lemma to the case when $M$ is a mapping torus. Then, we obtain $\beta_{\sigma, x}(\varphi)=B_{\alpha} \in \mathbf{Z}_{8}$, for $\varphi \in \mathcal{M}_{g, 1}[2]$ and $x \in H_{1}\left(\Sigma_{g} ; \mathbf{Z}_{2}\right)$.

\subsection{Heap's homomorphism}

In this subsection, we review the homomorphism $\omega_{\sigma, y}: \mathcal{I}_{g, 1} \rightarrow \mathbf{Z}_{2}$ defined by Heap $[6]$, and show that the homomorphism $\beta_{\sigma, x}$ defined in Subsection 3.3 is the extension of $\omega_{\sigma, y}$ to the level 2 mapping class group.

First we define a spin manifold $M^{\prime}=M_{\varphi}^{\prime}$. For $\sigma \in \operatorname{spin}\left(\Sigma_{g}\right)$ and $\varphi \in \mathcal{M}_{g, 1}[2]$, endow the spin structure $\theta(\sigma)$ on the mapping torus $M:=M_{\varphi}$. Denote by $M^{\prime}=\left(M-N\left(c_{0}\right) \times S^{1}\right) \cup\left(\partial N\left(c_{0}\right) \times D^{2}\right)$ the manifold obtained by the elementary surgery on $N\left(c_{0}\right) \times S^{1} \subset M$. We can choose the spin structure of $\partial N\left(c_{0}\right) \times D^{2}$ so that it induces in the boundary $\partial N\left(c_{0}\right) \times S^{1}$ the spin structure induced by $\theta(\sigma)$. Hence, the elementary surgery is compatible with the spin structure, and $M^{\prime}$ has the induced spin structure.

Next, we define Heap's homomorphism $\omega_{\sigma, y}$. For a group $G$, denote the spin bordism group of $K(G, 1)$ space by $\Omega_{*}^{\text {spin }}(G):=\Omega_{*}^{\text {spin }}(K(G, 1))$. Note that if $\varphi \in \mathcal{I}_{g, 1}$, we have $H_{1}\left(\Sigma_{g} ; \mathbf{Z}\right) \cong H_{1}\left(M^{\prime} ; \mathbf{Z}\right)$. Hence, we have the canonical homomorphism $\pi_{1}\left(M^{\prime}\right) \rightarrow H_{1}\left(\Sigma_{g} ; \mathbf{Z}\right)$. Let $f: M^{\prime} \rightarrow K\left(H_{1}\left(\Sigma_{g} ; \mathbf{Z}\right), 1\right)$ be a continuous map corresponding to this homomorphism. This map induces the homomorphism

$$
\eta_{\sigma, 2}: \mathcal{I}_{g, 1} \rightarrow \Omega_{3}^{s p i n}\left(H_{1}\left(\Sigma_{g} ; \mathbf{Z}\right)\right)
$$

which maps $\varphi \in \mathcal{I}_{g, 1}$ to $\left[\left(f, M^{\prime}\right)\right]$. In the same fashion, if $\varphi \in \mathcal{M}_{g, 1}[2]$, we have $H_{1}\left(\Sigma_{g} ; \mathbf{Z}_{2}\right) \cong H_{1}\left(M^{\prime} ; \mathbf{Z}_{2}\right)$. Let

$$
\eta_{\sigma, 2}[2]: \mathcal{M}_{g, 1}[2] \rightarrow \Omega_{3}^{s p i n}\left(H_{1}\left(\Sigma_{g} ; \mathbf{Z}_{2}\right)\right)
$$

be the homomorphism induced by $\pi_{1}\left(M^{\prime}\right) \rightarrow H_{1}\left(\Sigma_{g} ; \mathbf{Z}_{2}\right)$.

For $y \in H^{1}\left(\Sigma_{g} ; \mathbf{Z}\right)=\operatorname{Hom}\left(H_{1}\left(\Sigma_{g} ; \mathbf{Z}\right), \mathbf{Z}\right)$, we have the commutative diagram

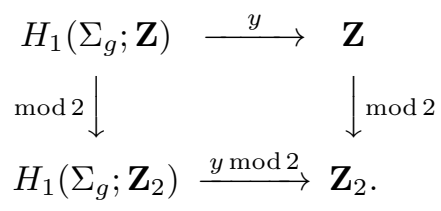

which induces the commutative diagram

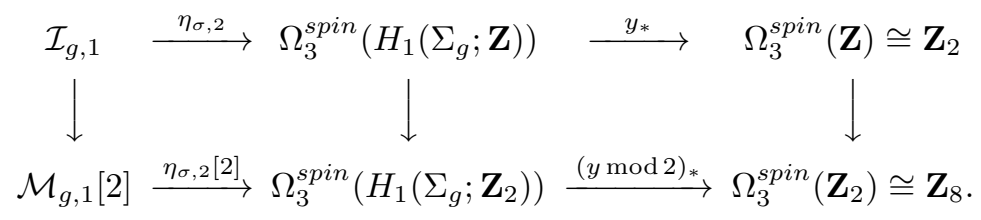

Then, Heap's homomorphism

$$
\omega_{\sigma, y}: \mathcal{I}_{g, 1} \rightarrow \mathbf{Z}_{2}
$$

is defined by $\omega_{\sigma, y}=y_{*} \eta_{\sigma, 2}$ for $y \in H_{1}\left(\Sigma_{g} ; \mathbf{Z}\right)$. 
Lemma 3.7. For $\sigma \in \operatorname{spin}\left(\Sigma_{g}\right), y \in H_{1}\left(\Sigma_{g} ; \mathbf{Z}\right)$ and $\psi \in \mathcal{I}_{g, 1}$,

$$
\beta_{\sigma, y \bmod 2}(\psi)=4 \omega_{\sigma, y}(\psi) \in \mathbf{Z}_{8}
$$

Proof. First, we explain the isomorphisms $\Omega_{3}^{\text {spin }}(\mathbf{Z}) \cong \mathbf{Z}_{2}$ and $\Omega_{3}^{\text {spin }}\left(\mathbf{Z}_{2}\right) \cong \mathbf{Z}_{8}$ in more detail.

For an $\left[\left(f, M^{\prime}\right)\right] \in \Omega_{3}^{s p i n}\left(S^{1}\right)$, choose a closed oriented surface $F_{y} \subset M^{\prime}$ which represents the Poincaré dual of the pullback $y:=f^{*} c \in H^{1}(M ; \mathbf{Z})$ of a generator $c \in H^{1}\left(S^{1} ; \mathbf{Z}\right)$. Then $F_{y}$ has the spin structure $\sigma_{y}$ induced by $\sigma \in \operatorname{spin}(M)$. For an oriented compact spin surface $F$, denote by $\operatorname{Arf}(\sigma)$ the Arf invariant of $\sigma \in \operatorname{spin}(F)$. By the Atiyah-Hirzebruch spectral sequence, the homomorphism

$$
\Omega_{3}^{\text {spin }}(\mathbf{Z}) \cong \Omega_{2}^{\text {spin }} \cong \mathbf{Z}_{2}
$$

defined by $\left[\left(f, M^{\prime}\right)\right] \mapsto \operatorname{Arf}\left(\sigma_{y}\right)$ is isomorphic. This homomorphism does not depend on the choice of the generator $c$.

Similarly, for $\left[\left(f, M^{\prime}\right)\right] \in \Omega_{3}^{\text {spin }}\left(\mathbf{Z}_{2}\right)$, choose a closed surface $F_{x} \subset M^{\prime}$ which represents the Poincaré dual of $x:=f^{*} w_{1} \in H^{1}\left(M^{\prime} ; \mathbf{Z}_{2}\right)$ of the Stiefel-Whitney class $\omega_{1} \in H^{1}\left(\mathbf{R P}^{\infty} ; \mathbf{Z}_{2}\right)$. Then, $F_{x} \subset M^{\prime}$ has the pin ${ }^{-}$ structure $\alpha_{x}$ induced from the spin structure of $M^{\prime}$. Then, there is an well-known isomorphism

$$
\Omega_{3}^{\text {spin }}\left(\mathbf{Z}_{2}\right) \rightarrow \Omega_{2}^{\text {pin }^{-}}
$$

given by $\left[\left(M^{\prime}, f\right)\right] \mapsto\left[F_{x}, \alpha_{x}\right]$. Under the isomorphism

$$
\Omega_{2}^{\text {pin }} \cong \mathbf{Z}_{8}
$$

$\left[F_{x}, \alpha_{x}\right]$ maps to $B_{\alpha_{x}}$.

Next, we prove $\beta_{\sigma, x}(\varphi)=x_{*} \eta_{\sigma, 2}[2](\varphi)$ for $\varphi \in \mathcal{M}_{g, 1}[2]$ and $x \in H_{1}\left(\Sigma_{g} ; \mathbf{Z}_{2}\right)$. Consider $x$ as an element of $H^{1}\left(M_{\varphi} ; \mathbf{Z}_{2}\right)$ under the inclusion $H_{1}\left(\Sigma_{g} ; \mathbf{Z}_{2}\right) \cong H^{1}\left(\Sigma_{g} ; \mathbf{Z}_{2}\right) \cong H^{1}\left(M_{\psi} ; \mathbf{Z}_{2}\right)$. We can choose a surface $F_{x} \subset$ $M-\left(N\left(c_{0}\right) \times S^{1}\right)$ which represents the Poincaré dual of $x$ with a pin ${ }^{-}$structure $\alpha_{x}$. Then, we have

$$
\beta_{\sigma, x}(\varphi)=B_{\alpha_{x}}=x_{*} \eta_{\sigma, 2}[2](\varphi) .
$$

By the definition of the Brown invariant, we see that the homomorphism $\Omega_{3}^{\text {spin }}(\mathbf{Z}) \rightarrow \Omega_{3}^{\text {spin }}\left(\mathbf{Z}_{2}\right) \cong \mathbf{Z}_{8}$ is written by 4 times the Arf invariant of a spin structure of the surface $F_{y}$. By the commutative diagram, we have $\beta_{\sigma, y \bmod 2}(\psi)=4 \omega_{\sigma, y}(\psi) \in \mathbf{Z}_{8}$ for $\psi \in \mathcal{I}_{g, 1}$ and $y \in H_{1}\left(\Sigma_{g} ; \mathbf{Z}\right)$.

\subsection{The value of $\beta_{\sigma, x}$}

Humphries ([7] p.314 Proposition 2.1) shows that the level 2 mapping class group $\mathcal{M}_{g, r}[2]$ is generated by the square of the Dehn twists along all non-separating simple closed curve when $g \geq 3$. We will compute the value of the homomorphism $\beta_{\sigma}$ defined in Subsection 3.3 on the generators of $\mathcal{M}_{g, 1}[2]$, using the Brown invariant. For $x \in H_{1}\left(\Sigma_{g} ; \mathbf{Z}_{2}\right)$, define the map $i_{x}: H_{1}\left(\Sigma_{g} ; \mathbf{Z}_{2}\right) \rightarrow \mathbf{Z}_{8}$ by

$$
i_{x}(y)= \begin{cases}1 & \text { if } x \cdot y \equiv 1 \bmod 2 \\ 0 & \text { if } x \cdot y \equiv 0 \bmod 2\end{cases}
$$

Note that this is not a homomorphism. For $\sigma \in \operatorname{spin}\left(\Sigma_{g}\right)$, denote by $q_{\sigma}: H_{1}\left(\Sigma_{g} ; \mathbf{Z}_{2}\right) \rightarrow \mathbf{Z}_{2}$ the quadratic function of $\sigma$.

Proposition 3.8. Let $C$ be a non-separating simple closed curve in $\Sigma_{g}-N\left(c_{0}\right)$. Then we have

$$
\beta_{\sigma}\left(t_{C}^{2}\right)=(-1)^{q_{\sigma}(C)} i_{[C]} \in \operatorname{Map}\left(H_{1}\left(\Sigma_{g} ; \mathbf{Z}_{2}\right), \mathbf{Z}_{8}\right) .
$$




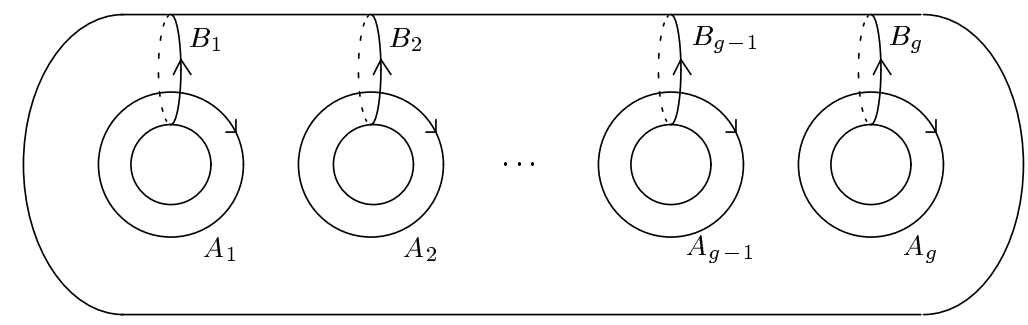

Figure 2: the symplectic basis

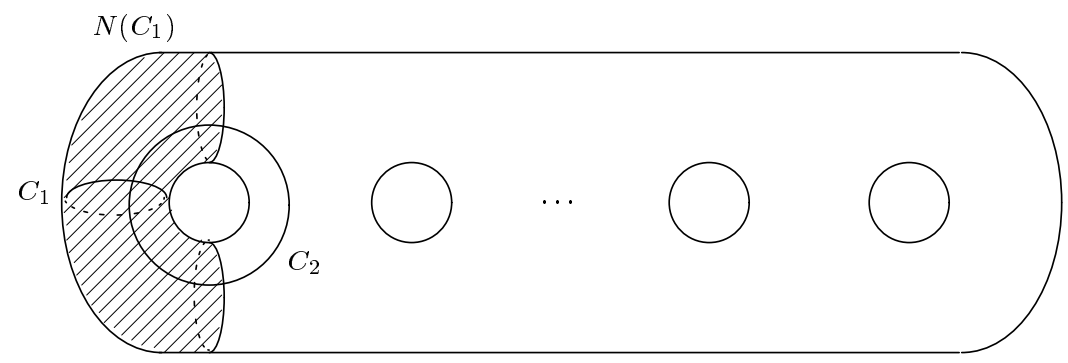

Figure 3: the neighborhood $N\left(C_{1}\right)$

Proof. We denote the symplectic basis $\left\{A_{i}, B_{i}\right\}_{i=1}^{g}$ represented by the simple closed curves in Figure 2 Choose the oriented simple closed curves $C_{1}$ and $C_{2}$ as described in Figure 3 . For any non-separating simple closed curve $C$, if we choose a mapping class $\varphi \in \mathcal{M}_{g, 1}$ such that $\varphi\left(C_{1}\right)=C$, we have

$$
\begin{aligned}
\beta_{\sigma, x}\left(t_{C}^{2}\right) & =\beta_{\sigma, x}\left(\varphi t_{C_{1}}^{2} \varphi^{-1}\right) \\
& =\left(R\left(M_{\varphi t_{C_{1}} \varphi^{-1}}^{2}, \theta(\sigma)\right)-R\left(M_{\varphi t_{C_{1}}^{2} \varphi^{-1}}, \theta(\sigma+x)\right)\right) / 2 \\
& =\left(R\left(M_{t_{C_{1}}^{2}}, \theta\left(\varphi^{*} \sigma\right)\right)-R\left(M_{t_{C_{1}}^{2}}, \theta\left(\varphi^{*} \sigma+\varphi_{*}^{-1}(x)\right)\right)\right) / 2 \\
& =\beta_{\varphi^{*} \sigma, \varphi_{*}^{-1}(x)}\left(t_{C_{1}}^{2}\right) .
\end{aligned}
$$

Hence It suffices to show that $\beta_{\sigma}\left(t_{C_{1}}^{2}\right)=(-1)^{q_{\sigma}\left(C_{1}\right)} i_{\left[C_{1}\right]}$. Let $M:=M_{t_{C_{1}}^{2}}$ denote a mapping torus.

First, we calculate the value $\beta_{\sigma, A_{1}+B_{1}}\left(t_{C_{1}}^{2}\right)$. Consider the compact 3-manifold $M_{1}:=N\left(C_{1}\right) \times I / \sim \subset M$. Choose the compact surface $F_{1} \subset M_{1}$ as shown in Figure 4. For the $\operatorname{arc} r=C_{2} \cap\left(\Sigma_{g}-\operatorname{Int} N\left(C_{1}\right)\right)$ as in Figure 3. denote another subsurface $F_{2}:=r \times S^{1} \subset M$. Then, the surface $F:=F_{1} \cup F_{2}$ represents the Poincaré dual of the homology class $A_{1}+B_{1} \in H_{1}\left(\Sigma_{g} ; \mathbf{Z}_{2}\right)=H^{1}\left(\Sigma_{g} ; \mathbf{Z}_{2}\right) \subset H^{1}\left(M ; \mathbf{Z}_{2}\right)$. Let $\alpha$ denote the pin ${ }^{-}$structure of $F$ induced by the spin structure of $M$. By Lemma 3.6 proved by Turaev, the value $\beta_{\sigma, A_{1}+B_{1}}\left(t_{C_{1}}^{2}\right)$ equals to the Brown invariant $B_{\alpha}$. Hence we investigate the quadratic enhancement $\hat{q}_{\alpha}: H_{1}\left(F ; \mathbf{Z}_{2}\right) \rightarrow \mathbf{Z}_{4}$ of the pin $^{-}$structure of $F$. Pick the generator $x, y, z$ of $H_{1}\left(F ; \mathbf{Z}_{2}\right)=\mathbf{Z}_{2} \oplus \mathbf{Z}_{2} \oplus \mathbf{Z}_{2}$ as in Figure 4. We may assume $x=A_{1}, y=B_{1}, z=\left[S^{1}\right] \in H_{1}(M ; \mathbf{Z})$. Then, we have

$$
\begin{gathered}
\hat{q}_{\alpha}(x)=-1+2 q_{\sigma}\left(A_{1}\right), \hat{q}_{\alpha}(y)=1+2 q_{\sigma}\left(B_{1}\right), \hat{q}_{\alpha}(z)=0, \\
\hat{q}_{\alpha}(x+y)=2+2 q_{\sigma}\left(A_{1}\right)+2 q_{\sigma}\left(B_{1}\right), \hat{q}_{\alpha}(y+z)=1+2 q_{\sigma}\left(B_{1}\right), \hat{q}_{\alpha}(z+x)=1+2 q_{\sigma}\left(A_{1}\right), \\
\hat{q}_{\alpha}(x+y+z)=2 q_{\sigma}\left(A_{1}\right)+2 q_{\sigma}\left(B_{1}\right) .
\end{gathered}
$$




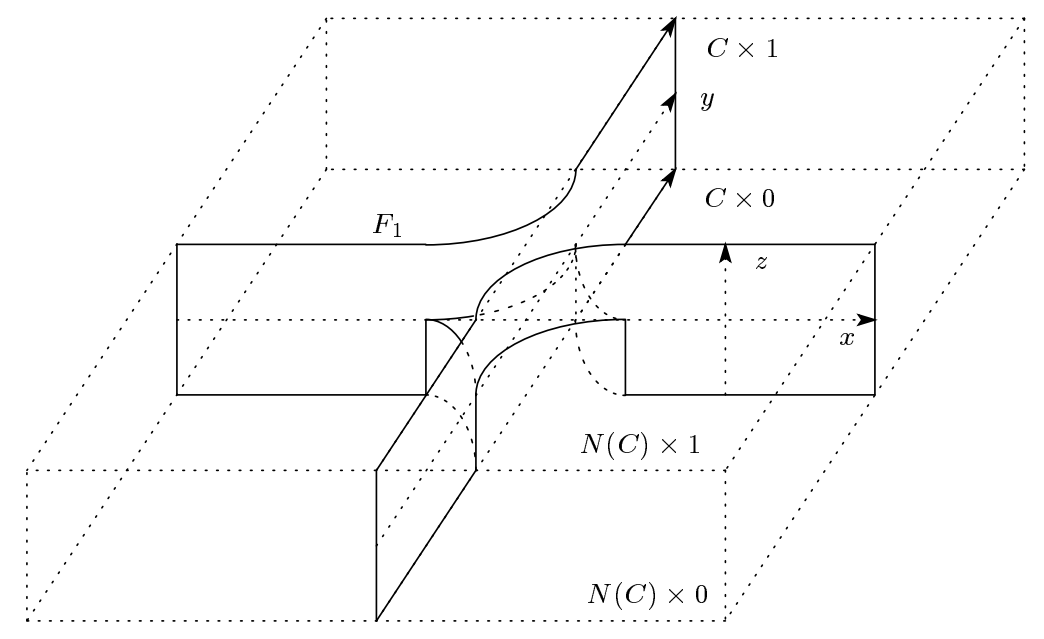

Figure 4: the surface $F_{1} \subset M_{1}$

Hence the Brown invariant $B_{\alpha}$ satisfies

$$
\begin{aligned}
\sqrt{\left|H_{1}\left(F ; \mathbf{Z}_{2}\right)\right|} \exp \left(2 \pi \sqrt{-1} B_{\alpha} / 8\right) & =\sum_{x \in H_{1}\left(F ; \mathbf{Z}_{2}\right)} \exp \left(2 \pi \sqrt{-1} \hat{q}_{\alpha}(x) / 4\right) \\
& =2 \exp \left(2 \pi \sqrt{-1}\left(2 q_{\sigma}\left(B_{1}\right)+1\right) / 4\right)+2 .
\end{aligned}
$$

Hence we have $\beta_{\sigma, A_{1}+B_{1}}\left(t_{C_{1}}^{2}\right)=B_{\alpha}=(-1)^{q_{\sigma}\left(B_{1}\right)}$.

Next, we show that $\beta_{\sigma, x}\left(t_{C_{1}}^{2}\right)=0$ for $x \in\left\langle B_{1}, A_{2}, B_{2} \cdots, A_{g}, B_{g}\right\rangle$. Choose the simple closed curve $C_{x} \subset \Sigma_{g}-$ $N\left(C_{1}\right)$ which represents $x \in H_{1}\left(\Sigma_{g} ; \mathbf{Z}_{2}\right)$. Denote the subsurface $F^{\prime}:=C_{x} \times S^{1} \subset M$. This subsurface represents the Poincaré dual of $x \in H_{1}\left(\Sigma_{g} ; \mathbf{Z}_{2}\right)=H^{1}\left(\Sigma_{g} ; \mathbf{Z}_{2}\right) \subset H^{1}\left(M ; \mathbf{Z}_{2}\right)$ in $M$. Choose a generator $x^{\prime}:=\left[C_{x} \times 0\right]$, $y^{\prime}:=\left[c \times S^{1}\right]$ of $H_{1}\left(F^{\prime} ; \mathbf{Z}_{2}\right)=\mathbf{Z}_{2} \oplus \mathbf{Z}_{2}$, where $c \in C_{x}$ is a point. Then, $F^{\prime}$ is orientable and the spin structure of $F^{\prime}$ is induced by that of $M$. We denote this spin structure by $\sigma^{\prime}$. Since spin group naturally injects into pin ${ }^{-}$ group, we can consider $\sigma^{\prime}$ as the pin ${ }^{-}$structure of $F^{\prime}$. Then, the quadratic enhancement $\hat{q}_{\sigma^{\prime}}$ is equal to twice the quadratic function $q_{\sigma^{\prime}}$. Hence we have

$$
\hat{q}_{\sigma^{\prime}}\left(x^{\prime}\right)=2 q_{\sigma^{\prime}}(x)=2 q_{\sigma}(x), \hat{q}_{\sigma^{\prime}}\left(y^{\prime}\right)=0 .
$$

This shows that $\beta_{\sigma, x}\left(t_{C_{1}}^{2}\right)=B_{\sigma^{\prime}}=0$.

Finally, we prove $\beta_{\sigma, A_{1}+x}\left(t_{C_{1}}^{2}\right)=\beta_{\sigma, A_{1}}\left(t_{C_{1}}^{2}\right)$. We have

$$
\begin{aligned}
\beta_{\sigma, A_{1}+x}\left(t_{C_{1}}^{2}\right) & =\left(R(M, \sigma)-R\left(M, \sigma+A_{1}+x\right)\right) / 2 \\
& =\left(R(M, \sigma)-R\left(M, \sigma+A_{1}\right)\right) / 2+\left(R\left(M, \sigma+A_{1}\right)-R\left(M, \sigma+A_{1}+x\right)\right) / 2 \\
& =\beta_{\sigma, A_{1}}\left(t_{C_{1}}^{2}\right)+\beta_{\sigma+A_{1}, x}\left(t_{C_{1}}^{2}\right) .
\end{aligned}
$$

Since we have $\beta_{\sigma, A_{1}}\left(t_{C_{1}}^{2}\right)=0$, it follows that $\beta_{\sigma, A_{1}+x}\left(t_{C_{1}}^{2}\right)=\beta_{\sigma, A_{1}}\left(t_{C_{1}}^{2}\right)$.

Thus, for all $x \in H_{1}\left(\Sigma_{g} ; \mathbf{Z}_{2}\right)$, we have

$$
\beta_{\sigma, x}\left(t_{C_{1}}^{2}\right)=(-1)^{q_{\sigma}\left(C_{1}\right)} i_{\left[C_{1}\right]}(x) .
$$




\section{Proof of Theorem 1.2}

We calculate the order of the homology group $H_{1}\left(\mathcal{M}_{g, 1}[2] ; \mathbf{Z}\right)$ in Subsections 4.2 and 4.3 , Using these results, we will prove Theorem 1.2. We also determine the abelianization of the level 2 mapping class group for closed surfaces.

\subsection{A homomorphism $\Phi: \mathbf{Z}\left[S_{d}\right] \rightarrow H_{1}\left(\mathcal{M}_{g, r}[d] ; \mathbf{Z}\right)$}

For a module $K=\mathbf{Z}, \mathbf{Z}_{d}$, we denote by $H_{1}\left(\Sigma_{g, r} ; K\right)^{\text {pri }}$ the set of primitive elements in $H_{1}\left(\Sigma_{g, r} ; K\right)$. Let

$$
S_{d}:=H_{1}\left(\Sigma_{g, r} ; \mathbf{Z}_{d}\right)^{\text {pri }} /\{ \pm 1\} .
$$

In this subsection, we define the homomorphism $\Phi: \mathbf{Z}\left[S_{d}\right] \rightarrow H_{1}\left(\mathcal{M}_{g, r}[d] ; \mathbf{Z}\right)$. In particular, this homomorphism is surjective when $d=2$.

The level $d$ mapping class group acts on the set of isotopy classes of non-separating simple closed curves. We will prove that $S_{d}$ corresponds bijectively to the orbit space of this action. Note that any element of $H_{1}\left(\Sigma_{g, r} ; \mathbf{Z}\right)^{p r i}$ is known to be represented by a simple closed curve.

Lemma 4.1. The mod $d$ reduction homomorphism $H_{1}\left(\Sigma_{g, r} ; \mathbf{Z}\right)^{\text {pri }} \rightarrow H_{1}\left(\Sigma_{g, r} ; \mathbf{Z}_{d}\right)^{\text {pri }}$ is surjective.

Proof. For $v_{d} \in H_{1}\left(\Sigma_{g, r} ; \mathbf{Z}_{d}\right)^{\text {pri }}$, choose $v \in H_{1}\left(\Sigma_{g, r} ; \mathbf{Z}\right)$ which satisfies $v \bmod d=v_{d} \in H_{1}\left(\Sigma_{g, r} ; \mathbf{Z}_{d}\right)^{\text {pri }}$. If $v$ is not primitive, there exists an integer $k \geq 2$ and a primitive element $w \in H_{1}\left(\Sigma_{g, r} ; \mathbf{Z}\right)^{\text {pri }}$ such that $v=k w$. Since $v_{d}$ is primitive, $k$ and $d$ are coprime. Then, there exist integers $k^{\prime}, d^{\prime} \in \mathbf{Z}$ such that $k k^{\prime}+d d^{\prime}=1$. Choose $w^{\prime} \in H_{1}\left(\Sigma_{g, r} ; \mathbf{Z}\right)^{p r i}$ such that $w \cdot w^{\prime}=1$. We have

$$
\left(v+d w^{\prime}\right) \cdot\left(-d^{\prime} w+k^{\prime} w^{\prime}\right)=k k^{\prime}+d d^{\prime}=1
$$

Hence, $v+d w^{\prime} \in H_{1}\left(\Sigma_{g, r} ; \mathbf{Z}\right)$ is primitive and $v+d w^{\prime} \bmod d=v_{d} \in H_{1}\left(\Sigma_{g, r} ; \mathbf{Z}_{d}\right)$.

Lemma 4.2. Let $C_{1}, C_{1}^{\prime} \subset \Sigma_{g, r}$ be non-separating simple closed curves such that $\left[C_{1}\right]=\left[C_{1}^{\prime}\right] \in H_{1}\left(\Sigma_{g, r} ; \mathbf{Z}_{d}\right) /\{ \pm 1\}$. Then, there exists a mapping class $[f] \in \mathcal{M}_{g, r}[d]$ such that $f\left(C_{1}\right)=C_{1}^{\prime}$.

Proof. Fix orientations of $C_{1}$ and $C_{1}^{\prime}$ so that $\left[C_{1}\right]=\left[C_{1}^{\prime}\right] \in H_{1}\left(\Sigma_{g, r} ; \mathbf{Z}_{d}\right)$. Denote $u:=\left(\left[C_{1}^{\prime}\right]-\left[C_{1}\right]\right) / d \in$ $H_{1}\left(\Sigma_{g, r} ; \mathbf{Z}\right)$. Choose the simple closed curve $C_{2}$ which intersects $C_{1}$ transversely at one point. Since $\left[C_{1}^{\prime}\right] \in$ $H_{1}\left(\Sigma_{g, r} ; \mathbf{Z}\right)$ is primitive, there exists $v \in H_{1}\left(\Sigma_{g, r} ; \mathbf{Z}\right)$ which satisfies $\left[C_{1}^{\prime}\right] \cdot v=-u \cdot\left[C_{2}\right]$. If we put $\alpha_{2}^{\prime}:=\left[C_{2}\right]+d v$, we have

$$
\begin{aligned}
{\left[C_{1}^{\prime}\right] \cdot \alpha_{2}^{\prime} } & =\left[C_{1}^{\prime}\right] \cdot\left(\left[C_{2}\right]+d v\right) \\
& =\left(d u+\left[C_{1}\right]\right) \cdot\left[C_{2}\right]+d\left[C_{1}^{\prime}\right] \cdot v \\
& =d u \cdot\left[C_{2}\right]+1+d\left[C_{1}^{\prime}\right] \cdot v \\
& =1 .
\end{aligned}
$$

In particular, the element $\alpha_{2}^{\prime}$ is primitive. Hence there exists $C_{2}^{\prime}$ such that $\left[C_{2}^{\prime}\right]=\alpha_{2}^{\prime}$, and intersect $C_{1}^{\prime}$ transversely in one point.

Choose a diffeomorphism $f: \Sigma_{g, r} \rightarrow \Sigma_{g, r}$ which satsifies $f\left(C_{1}\right)=C_{1}^{\prime}, f\left(C_{2}\right)=C_{2}^{\prime},\left.f\right|_{\partial \Sigma_{g, r}}=i d_{\partial \Sigma_{g, r}}$. Denote by $\left\{Y_{i}\right\}_{i=2}^{2 g-2}$ the homology class of $H_{1}\left(\Sigma_{g, r} ; \mathbf{Z}\right)$ such that $\left\{\left[C_{1}\right],\left[C_{2}\right]\right\} \cup\left\{Y_{i}\right\}_{i=1}^{2 g-2}$ makes the symplectic basis. Since we have $f_{*}\left(\left[C_{i}\right]\right) \equiv\left[C_{i}\right] \bmod d$ for $i=1,2$, The symplectic action of $f$ on $H_{1}\left(\Sigma_{g, r} ; \mathbf{Z}_{d}\right)$ induces the action on $\bigoplus_{i=1}^{2 g-2} \mathbf{Z}_{d} Y_{i}$. For an closed tubular neighborhood $N\left(C_{i}\right)$ of $C_{i}$, denote the surface $F:=\Sigma_{g, r}-\left(\cup \operatorname{Int} N\left(C_{i}\right)\right)$. Here, the action of the mapping class group $\mathcal{M}_{g-1, r+1}$ of $F$ on $H_{1}\left(F ; \mathbf{Z}_{d}\right) / \operatorname{Im}\left(H_{1}\left(\partial F ; \mathbf{Z}_{d}\right) \rightarrow H_{1}\left(F ; \mathbf{Z}_{d}\right)\right)$ induces 
the surjective homomorphism $\mathcal{M}_{g-1, r+1} \rightarrow \mathrm{Sp}(2 g-2 ; \mathbf{Z}) \rightarrow \operatorname{Sp}\left(2 g-2 ; \mathbf{Z}_{d}\right)$. Hence there exists $g \in \operatorname{Diff}(F, \partial F)$ such that

$$
g_{*}\left(Y_{i}\right)=f_{*}^{-1}\left(Y_{i}\right) \in H_{1}\left(F ; \mathbf{Z}_{d}\right) .
$$

It is easy to see that $\left[f\left(g \cup i d_{\amalg N\left(C_{i}\right)}\right)\right] \in \mathcal{M}_{g, r}[d]$ is the desired mapping class.

By Lemma 4.1, every element of $S_{d}$ is represented by a simple closed curve. By Lemma 4.2, $S_{d}$ corresponds to the orbit space of the action of $\mathcal{M}_{g, r}[d]$ on isotopy classes of non-separating simple closed curves.

Now, we define the homomorphism $\mathbf{Z}\left[S_{d}\right] \rightarrow H_{1}\left(\mathcal{M}_{g, r}[d] ; \mathbf{Z}\right)$. Denote by $t_{C} \in \mathcal{M}_{g, r}$ the Dehn twist along a simple closed curve $C \subset \Sigma_{g, r}$. By Lemmas 4.1 and 4.2, we can define the map $\Phi: S_{d} \rightarrow H_{1}\left(\mathcal{M}_{g, r}[d] ; \mathbf{Z}\right)$ by $\Phi([C])=\langle[C]\rangle:=\left[t_{C}^{d}\right]$. Extend this map to a homomorphism of $\mathbf{Z}$-module

$$
\Phi_{d}: \mathbf{Z}\left[S_{d}\right] \rightarrow H_{1}\left(\mathcal{M}_{g, r}[d] ; \mathbf{Z}\right) .
$$

We consider the case when $d=2$. Then, we have $S_{2}=H_{1}\left(\Sigma_{g, r} ; \mathbf{Z}_{2}\right)-0$. Define $\Phi_{2}([0]):=0$ and extend $\Phi_{2}$ to

$$
\Phi:=\Phi_{2}: \mathbf{Z}\left[H_{1}\left(\Sigma_{g, r} ; \mathbf{Z}_{2}\right)\right] \rightarrow H_{1}\left(\mathcal{M}_{g, r}[2] ; \mathbf{Z}\right) .
$$

Lemma 4.3. The homomorphism $\Phi$ is surjective, and factors through $\mathbf{Z}_{8}\left[H_{1}\left(\Sigma_{g, r} ; \mathbf{Z}_{2}\right)\right]$.

Proof. Humphries [7 proved that the level 2 mapping class group is generated by Dehn twists along nonseparating curves. Hence, $\Phi$ is surjective. Denote by $H_{1}\left(\mathcal{I}_{g, r} ; \mathbf{Z}\right)_{\mathcal{M}_{g, r}[2]}$ the coinvariant of the action of $\mathcal{M}_{g, r}[2]$ on $H_{1}\left(\mathcal{I}_{g, r} ; \mathbf{Z}\right)$. Consider the exact sequence

$$
H_{1}\left(\mathcal{I}_{g, r} ; \mathbf{Z}\right)_{\mathcal{M}_{g, r}[2]} \longrightarrow H_{1}\left(\mathcal{M}_{g, r}[2] ; \mathbf{Z}\right) \longrightarrow H_{1}\left(\Gamma_{g}[2] ; \mathbf{Z}\right) \longrightarrow 0 .
$$

The coinvariant $H_{1}\left(\mathcal{I}_{g, r} ; \mathbf{Z}\right)_{\mathcal{M}_{g, r}[2]}$ is proved to be a $\mathbf{Z}_{2}$-module in Johnson [13] Theorems 1 and 4, and we proved that $H_{1}\left(\Gamma_{g}[2] ; \mathbf{Z}\right)$ is a $\mathbf{Z}_{4}$-module in Section 2 . Hence $H_{1}\left(\mathcal{M}_{g, r}[2] ; \mathbf{Z}\right)$ is a $\mathbf{Z}_{8}$-module. This shows that $\Phi$ factors through the module $\mathbf{Z}_{8}\left[H_{1}\left(\Sigma_{g, r} ; \mathbf{Z}_{2}\right)\right]$.

\subsection{Upper bound of the order $\left|H_{1}\left(\mathcal{M}_{g, 1}[2] ; \mathbf{Z}\right)\right|$}

In this subsection, we examine the kernel of the inclusion homomorphism

$$
H_{1}\left(\mathcal{I}_{g, r} ; \mathbf{Z}\right)_{\mathcal{M}_{g, r}[2]} \rightarrow H_{1}\left(\mathcal{M}_{g, r}[2] ; \mathbf{Z}\right),
$$

and give an upper bound of the order of $H_{1}\left(\mathcal{M}_{g, 1}[2] ; \mathbf{Z}\right)$.

First, we review the $\mathbf{Z}_{2}$-module $B_{g, r}^{3}$ defined by Johnson[12]. We consider the commutative polynomial ring $R$ with coefficient $\mathbf{Z}_{2}$ in formal symbol $\bar{x}$ for $x \in H_{1}\left(\Sigma_{g, r} ; \mathbf{Z}\right)$. Denote by $J$ the ideal of this polynomial ring generated by

$$
\overline{x+y}-(\bar{x}+\bar{y}+x \cdot y), \quad \bar{x}^{2}-\bar{x},
$$

for $x, y \in H \otimes \mathbf{Z}_{2}$,

Denote by $R_{n}$ the module consisting of polynomials whose degrees are less than or equal to $n$. Define the module $B^{n}$ by

$$
B^{n}=\frac{R_{n}}{J \cap R_{n}},
$$

and denote

$$
B_{g, 1}^{3}:=B^{3} .
$$

Let $\left.A_{i}, B_{i}\right\}_{i=1}^{g}$ denote a symplectic basis defined in Proposition 3.8 , For the element $\alpha=\sum_{i=1}^{g} \bar{A}_{i} \bar{B}_{i} \in B^{2}$, define the homomorphism $B^{1} \rightarrow B_{g, 1}^{3}$ by $x \mapsto x \alpha$. Denote its cokernel by $B_{g}^{3}$. Johnson determined the $\operatorname{Sp}(2 g ; \mathbf{Z})$ module structure of $H_{1}\left(\mathcal{I}_{g, r} ; \mathbf{Z}\right)_{\mathcal{M}_{g, r}[2]}$. 
Theorem 4.4 (Johnson[13] Theorem 1, Theorem 4).

$$
H_{1}\left(\mathcal{I}_{g, r} ; \mathbf{Z}\right)_{\mathcal{M}_{g, r}[2]} \cong B_{g, r}^{3} .
$$

Next, we examine the kernel of $\iota: B_{g, r}^{3} \cong H_{1}\left(\mathcal{I}_{g, r} ; \mathbf{Z}\right)_{\mathcal{M}_{g, r}[2]} \rightarrow H_{1}\left(\mathcal{M}_{g, r}[2] ; \mathbf{Z}\right)$.

Lemma 4.5. For $r=0,1$,

$$
1 \in \operatorname{Ker} \iota \text {. }
$$

Proof. As in Figure 5, choose the simple closed curves $C_{1}, C_{2}, D_{1}$ so that $\left[C_{1}\right]=B_{1},\left[C_{2}\right]=A_{1}$. For $X \in$ $H_{1}\left(\Sigma_{g, r} ; \mathbf{Z}_{2}\right)$, we denote simply $\langle X\rangle:=\Phi(X)$. Then, by Lemma 12a in Johnson[12] and the chain relation, we

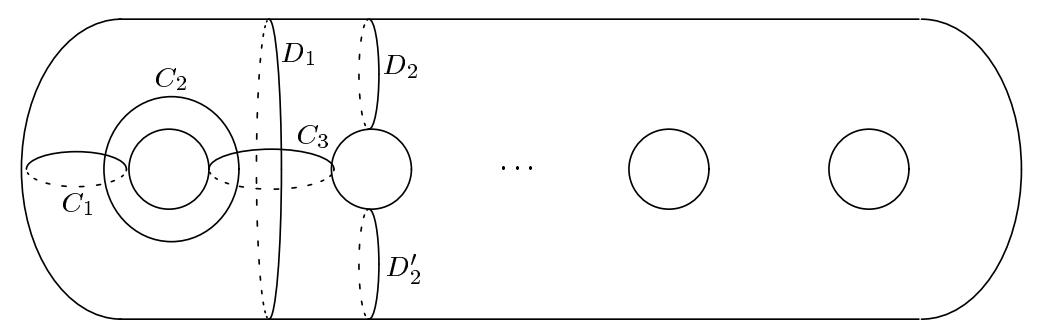

Figure 5: the curves

have

$$
\begin{aligned}
\iota\left(\bar{A}_{1} \bar{B}_{1}\right) & =\left[t_{D_{1}}\right] \\
& =\left[\left(t_{C_{1}} t_{C_{2}}\right)^{6}\right] \\
& =2\left[t_{C_{1}}^{2}\right]+2\left[t_{C_{2}}^{2}\right]+2\left[t_{C_{1}}^{-1} t_{C_{2}}^{2} t_{C_{1}}\right] \\
& =2\left\langle A_{1}\right\rangle+2\left\langle B_{1}\right\rangle+2\left\langle A_{1}+B_{1}\right\rangle .
\end{aligned}
$$

If we choose $\varphi \in \mathcal{M}_{g, r}$ such that $\varphi\left(A_{1}\right)=A_{1}, \varphi\left(B_{1}\right)=B_{1}+B_{2}$, we have

$$
\iota\left(\bar{A}_{1}\left(\overline{B_{1}+B_{2}}\right)\right)=2\left\langle A_{1}\right\rangle+2\left\langle B_{1}+B_{2}\right\rangle+2\left\langle A_{1}+B_{1}+B_{2}\right\rangle .
$$

In the same fashion, by Lemma $12 \mathrm{~b}$ in Johnson[12] and the chain relation, we have

$$
\begin{aligned}
\iota\left(\bar{A}_{1} \bar{B}_{1}\left(\overline{B_{2}}+1\right)\right) & =\left[t_{D_{2}} t_{D_{2}^{\prime}}^{-1}\right] \\
& =\left[\left(t_{C_{1}} t_{C_{2}} t_{C_{3}}\right)^{4}\right]-\left\langle B_{2}\right\rangle \\
& =\left\langle B_{1}\right\rangle+\left\langle A_{1}\right\rangle+\left\langle B_{1}+B_{2}\right\rangle+\left\langle A_{1}+B_{1}\right\rangle+\left\langle A_{1}+B_{1}+B_{2}\right\rangle+\left\langle A_{1}+B_{2}\right\rangle-\left\langle B_{2}\right\rangle .
\end{aligned}
$$

Since $\iota\left(2 \bar{A}_{1} \bar{B}_{1}\left(\overline{B_{2}}+1\right)\right)=0$, we have

$$
2\left\langle A_{1}+B_{1}+B_{2}\right\rangle=-2\left(\left\langle B_{1}\right\rangle+\left\langle A_{1}\right\rangle+\left\langle B_{1}+B_{2}\right\rangle+\left\langle A_{1}+B_{1}\right\rangle+\left\langle A_{1}+B_{2}\right\rangle-\left\langle B_{2}\right\rangle\right) .
$$

Put this into the equation (16), then we have

$$
\begin{aligned}
\iota\left(\bar{A}_{1}\left(\overline{B_{1}+B_{2}}\right)\right) & =2\left\langle A_{1}\right\rangle+2\left\langle B_{1}+B_{2}\right\rangle \\
& -2\left(\left\langle A_{1}\right\rangle+\left\langle B_{1}\right\rangle-\left\langle B_{2}\right\rangle+\left\langle A_{1}+B_{1}\right\rangle+\left\langle A_{1}+B_{2}\right\rangle+\left\langle B_{1}+B_{2}\right\rangle\right) \\
& =-2\left\langle B_{1}\right\rangle+2\left\langle B_{2}\right\rangle-2\left\langle A_{1}+B_{1}\right\rangle-2\left\langle A_{1}+B_{2}\right\rangle .
\end{aligned}
$$


By the equation (15) and (17),

$$
\begin{aligned}
\iota\left(\bar{A}_{1} \bar{B}_{2}\right) & =\iota\left(\bar{A}_{1}\left(\overline{B_{1}+B_{2}}\right)+\bar{A}_{1} \bar{B}_{1}\right) \\
& =2\left\langle A_{1}\right\rangle+2\left\langle B_{2}\right\rangle-2\left\langle A_{1}+B_{2}\right\rangle .
\end{aligned}
$$

If we choose $\varphi \in \mathcal{M}_{g, r}$ so that $\varphi_{*}\left(A_{1}\right)=A_{1}, \varphi_{*}\left(B_{2}\right)=A_{1}+B_{2}$, we have

$$
\iota\left(\bar{A}_{1}\left(\bar{A}_{1}+\bar{B}_{2}\right)\right)=2\left\langle A_{1}\right\rangle+2\left\langle A_{1}+B_{2}\right\rangle-2\left\langle B_{2}\right\rangle .
$$

Hence we obtain

$$
\begin{aligned}
\iota\left(\bar{A}_{1}\right) & =\iota\left(\bar{A}_{1}\left(\bar{A}_{1}+\bar{B}_{2}\right)-\bar{A}_{1} \overline{B_{2}}\right) \\
& =4\left\langle A_{1}\right\rangle .
\end{aligned}
$$

As we stated in the last subsection, $H_{1}\left(\mathcal{M}_{g, r}[2] ; \mathbf{Z}\right)$ is a $\mathbf{Z}_{8}$-module. Hence we have $8\left\langle A_{1}\right\rangle=8\left\langle B_{1}\right\rangle=0$. Therefore, we see that

$$
\begin{aligned}
\iota(1) & =\iota\left(\overline{A_{1}+B_{1}}-\bar{A}_{1}-\bar{B}_{1}\right) \\
& =4\left(\left\langle A_{1}+B_{1}\right\rangle-\left\langle A_{1}\right\rangle-\left\langle B_{1}\right\rangle\right) \\
& =4\left(\left\langle A_{1}+B_{1}\right\rangle+\left\langle A_{1}\right\rangle+\left\langle B_{1}\right\rangle\right) \\
& =\iota\left(2 \bar{A}_{1} \bar{B}_{1}\right)=0 \in H_{1}\left(\mathcal{M}_{g, r}[2] ; \mathbf{Z}\right) .
\end{aligned}
$$

By this lemma, we obtain the upper bound

$$
\left|H_{1}\left(\mathcal{M}_{g, 1}[2] ; \mathbf{Z}\right)\right| \leq\left|B_{g, 1}^{3} /\langle 1\rangle\right|\left|H_{1}\left(\Gamma_{g}[2] ; \mathbf{Z}\right)\right| .
$$

\subsection{Lower bound of the order $\left|H_{1}\left(\mathcal{M}_{g, 1}[2] ; \mathbf{Z}\right)\right|$}

In this subsection, we give a lower bound of the order of $H_{1}\left(\mathcal{M}_{g, 1}[2] ; \mathbf{Z}\right)$

$$
\left|H_{1}\left(\mathcal{M}_{g, 1}[2] ; \mathbf{Z}\right)\right| \geq\left|\mathbf{Z}_{8}^{2 g} \oplus \mathbf{Z}_{4}^{\left(\begin{array}{c}
2 g \\
2
\end{array}\right)} \oplus \mathbf{Z}_{2}^{\left(\begin{array}{c}
2 g \\
3
\end{array}\right)}\right| .
$$

Using this result we determine the abelianization $H_{1}\left(\mathcal{M}_{g, 1}[2] ; \mathbf{Z}\right)$.

For $\sigma \in \operatorname{spin}\left(\Sigma_{g}\right)$ and $\left\{x_{j}\right\}_{j=1}^{n} \subset H_{1}\left(\Sigma_{g, 1} ; \mathbf{Z}_{2}\right)$, define $\Delta_{\sigma}\left(x_{1}, x_{2}, \cdots, x_{n}\right)$ by

$$
\begin{aligned}
\Delta_{\sigma}\left(x_{1}, x_{2}, \cdots, x_{n}\right):=\sum_{j=1}^{n}(-1)^{q_{\sigma}\left(x_{j}\right)}\left[x_{j}\right] & +\sum_{1 \leq j<k \leq n}^{n}(-1)^{q_{\sigma}\left(x_{j}+x_{k}\right)}\left[x_{j}+x_{k}\right] \\
& +\cdots+(-1)^{q_{\sigma}\left(x_{1}+x_{2}+\cdots+x_{n}\right)}\left[x_{1}+x_{2}+\cdots+x_{n}\right] \in \mathbf{Z}_{8}\left[H_{1}\left(\Sigma_{g, 1} ; \mathbf{Z}_{2}\right)\right] .
\end{aligned}
$$

\section{Lemma 4.6.}

$$
\begin{aligned}
\Delta_{\sigma}\left(x_{1}, x_{2}, \cdots, x_{n-1}, x_{n}, x_{n+1}\right)= & \Delta_{\sigma}\left(x_{1}, x_{2}, \cdots, x_{n-1}, x_{n}+x_{n+1}\right)+\Delta_{\sigma}\left(x_{1}, x_{2}, \cdots, x_{n-1}, x_{n}\right) \\
& +\Delta_{\sigma}\left(x_{1}, x_{2}, \cdots, x_{n-1}, x_{n+1}\right)-2 \Delta_{\sigma}\left(x_{1}, x_{2}, \cdots, x_{n-1}\right) .
\end{aligned}
$$

Proof. For $X \in H_{1}\left(\Sigma_{g, r} ; \mathbf{Z}_{2}\right)$, denote

$$
\begin{aligned}
\Delta_{\sigma}^{X}\left(x_{1}, x_{2}, \cdots, x_{n}\right):=\sum_{j=1}^{n}(-1)^{q_{\sigma}\left(x_{j}+X\right)}\left[x_{j}+X\right]+ & \sum_{1 \leq j<k \leq n}^{n}(-1)^{q_{\sigma}\left(x_{j}+x_{k}+X\right)}\left[x_{j}+x_{k}+X\right] \\
& +\cdots+(-1)^{q_{\sigma}\left(x_{1}+x_{2}+\cdots+x_{n}+X\right)}\left[x_{1}+x_{2}+\cdots+x_{n}+X\right] .
\end{aligned}
$$


By the definition of $\Delta_{\sigma}$, we have

$$
\Delta_{\sigma}\left(x_{1}, x_{2}, \cdots, x_{n-1}, x_{n}, x_{n+1}\right)=\Delta_{\sigma}\left(x_{1}, x_{2}, \cdots, x_{n-1}, x_{n}\right)+\Delta_{\sigma}^{x_{n+1}}\left(x_{1}, x_{2}, \cdots, x_{n-1}, x_{n}\right)
$$

Similarly, we see that

$$
\begin{aligned}
\Delta_{\sigma}^{x_{n+1}}\left(x_{1}, x_{2}, \cdots, x_{n-1}, x_{n}\right)= & \Delta_{\sigma}^{x_{n+1}}\left(x_{1}, x_{2}, \cdots, x_{n-1}\right)+\Delta_{\sigma}^{x_{n}+x_{n+1}}\left(x_{1}, x_{2}, \cdots, x_{n-1}\right) \\
= & \Delta_{\sigma}\left(x_{1}, x_{2}, \cdots, x_{n-1}, x_{n+1}\right)-\Delta_{\sigma}\left(x_{1}, x_{2}, \cdots, x_{n-1}\right) \\
& +\Delta_{\sigma}\left(x_{1}, x_{2}, \cdots, x_{n-1}, x_{n}+x_{n+1}\right)-\Delta_{\sigma}\left(x_{1}, x_{2}, \cdots, x_{n-1}\right)
\end{aligned}
$$

Put this into the equation (18), then we obtain what we intended to prove.

Lemma 4.7. For $\left\{x_{j}\right\}_{j=1}^{n} \subset H_{1}\left(\Sigma_{g, 1} ; \mathbf{Z}_{2}\right)$ and $x \in H_{1}\left(\Sigma_{g} ; \mathbf{Z}_{2}\right)$,

$$
\beta_{\sigma} \Phi\left(\Delta_{\sigma}^{n}\left(x_{1}, x_{2}, \cdots, x_{n}\right)\right)(x)=2^{n-1} \prod_{j=1}^{n} i_{x_{j}}(x) .
$$

Proof. In Proposition 3.8 we proved that $\beta_{\sigma} \Phi\left((-1)^{q_{\sigma}(x)} x_{1}\right)(x)=i_{x_{1}}(x)$. Assume that for $n-1$ the equation holds. By the Lemma 4.6, we have

$$
\begin{aligned}
\beta_{\sigma} \Phi\left(\Delta_{\sigma}^{n}\left(x_{1}, x_{2}, \cdots, x_{n}, x_{n+1}\right)\right)(x) & =2^{n-1} \prod_{j=1}^{n-1} i_{x_{j}}(x)\left(i_{x_{n}+x_{n+1}}(x)+i_{x_{n}}(x)+i_{x_{n+1}}(x)-1\right) \\
& =2^{n} \prod_{j=1}^{n+1} i_{x_{j}}(x) .
\end{aligned}
$$

This proves the lemma.

Denote the homology classes $X_{n}$ by $X_{2 j-1}:=A_{j}$, and $X_{2 j}=B_{j}$ for $j=1,2, \cdots, g$. For convenience, we denote $X_{n+2 g}=X_{n}$ for $n=1,2, \cdots 2 g$. Define the surjective homomorphism

$$
\Psi: \operatorname{Map}\left(H_{1}\left(\Sigma_{g} ; \mathbf{Z}_{2}\right) ; \mathbf{Z}_{8}\right) \rightarrow \mathbf{Z}_{8}^{2 g} \oplus \mathbf{Z}_{8}^{\left(\begin{array}{c}
2 g \\
2
\end{array}\right)} \oplus \mathbf{Z}_{8}^{\left(\begin{array}{c}
2 g \\
3
\end{array}\right)}
$$

by

$$
\Psi(f):=\left(\left\{f\left(X_{i_{1}}\right)\right\}_{i_{1}=1}^{2 g},\left\{f\left(X_{i_{1}}+X_{i_{2}}\right)\right\}_{1 \leq i_{1} \leq i_{2} \leq 2 g},\left\{f\left(X_{i_{1}}+X_{i_{2}}+X_{i_{3}}\right)\right\}_{1 \leq i_{1} \leq i_{2} \leq i_{3} \leq 2 g}\right) .
$$

\section{Lemma 4.8 .}

$$
\operatorname{Im}\left(\Psi \beta_{\sigma}\right)=\mathbf{Z}_{8}^{2 g} \oplus 2 \mathbf{Z}_{8}^{\left(\begin{array}{c}
2 g \\
2
\end{array}\right)} \oplus 4 \mathbf{Z}_{8}^{\left(\begin{array}{c}
2 g \\
3
\end{array}\right)} .
$$

Proof. We examine the value of $\Psi \beta_{\sigma}$ on $\Phi\left(\Delta_{\sigma}\left(X_{i_{1}}, X_{i_{2}}, \cdots, X_{i_{n}}\right)\right) \in H_{1}\left(\mathcal{M}_{g, 1}[2] ; \mathbf{Z}\right)$, using Lemma 4.7

For $f=\beta_{\sigma} \Phi\left(\Delta_{\sigma}\left(X_{i_{1}}\right)\right)$ where $1 \leq i_{1} \leq 2 g$, we have

$$
f\left(X_{l}\right)=\left\{\begin{array}{l}
1, \text { if } X_{l}=X_{i_{1}+g}, \\
0, \text { otherwise, }
\end{array} \quad f\left(X_{l}+X_{m}\right)=f\left(X_{l}+X_{m}+X_{n}\right)=0 .\right.
$$

For $f=\beta_{\sigma} \Phi\left(\Delta_{\sigma}\left(X_{i_{1}}, X_{i_{2}}\right)\right)$ where $1 \leq i_{1}<i_{2} \leq 2 g$,

$$
\begin{gathered}
f\left(X_{l}\right)=0, \\
f\left(X_{l}+X_{m}\right)=\left\{\begin{array}{l}
2, \text { if }\left\{X_{l}, X_{m}\right\}=\left\{X_{i_{1}+g}, X_{i_{2}+g}\right\}, \\
0, \text { otherwise, }
\end{array} \quad f\left(X_{l}+X_{m}+X_{n}\right)=0 .\right.
\end{gathered}
$$


For $f=\beta_{\sigma} \Phi\left(\Delta_{\sigma}\left(X_{i_{1}}, X_{i_{2}}, X_{i_{3}}\right)\right)$ where $1 \leq i_{1}<i_{2}<i_{3} \leq 2 g$,

$$
\begin{gathered}
f\left(X_{l}\right)=f\left(X_{l}+X_{m}\right)=0, \\
f\left(X_{l}+X_{m}+X_{n}\right)=\left\{\begin{array}{l}
4, \text { if }\left\{X_{l}, X_{m}, X_{n}\right\}=\left\{X_{i_{1}+g}, X_{i_{2}+g}, X_{i_{3}+g}\right\} \\
0, \text { otherwise. }
\end{array}\right.
\end{gathered}
$$

We also have $\beta_{\sigma} \Phi\left(\Delta_{\sigma}\left(X_{i_{1}}, X_{i_{2}}, \cdots, X_{i_{n}}\right)\right)=0$ for $n \geq 4$.

Since $\Phi$ is surjective, we have determined the image of the homomorphism $\Psi \beta_{\sigma}$.

By this lemma, we obtain the lower bound

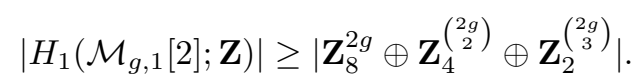

Now, we determine the abelianization $H_{1}\left(\mathcal{M}_{g, 1}[2] ; \mathbf{Z}\right)$ as a $\mathbf{Z}$-module.

Proposition 4.9. For $g \geq 3$,

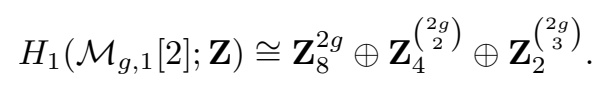

Proof. Denote by $\langle 1\rangle$ the cyclic group generated by $1 \in B_{g, r}^{3}$. We have

$$
\left|\mathbf{Z}_{8}^{2 g} \oplus \mathbf{Z}_{4}^{\left(\begin{array}{c}
2 g \\
2
\end{array}\right)} \oplus \mathbf{Z}_{2}^{\left(\begin{array}{c}
2 g \\
3
\end{array}\right)}\right| \leq\left|H_{1}\left(\mathcal{M}_{g, 1}[2] ; \mathbf{Z}\right)\right| \leq\left|B_{g, 1}^{3} /\langle 1\rangle\right|\left|H_{1}\left(\Gamma_{g}[2] ; \mathbf{Z}\right)\right| .
$$

By the definition of $B_{g, 1}^{3}$, it is easy to see that

$$
\left|B_{g, 1}^{3} /\langle 1\rangle\right|\left|H_{1}\left(\Gamma_{g}[2] ; \mathbf{Z}\right)\right|=\left|\mathbf{Z}_{8}^{2 g} \oplus \mathbf{Z}_{4}^{\left(\begin{array}{c}
2 g \\
2
\end{array}\right)} \oplus \mathbf{Z}_{2}^{\left(\begin{array}{c}
2 g \\
3
\end{array}\right)}\right| .
$$

By comparing the order of groups, we see that the surjective homomorphism

$$
\left.\left.\Psi \beta_{\sigma}: H_{1}\left(\mathcal{M}_{g, 1}[2] ; \mathbf{Z}\right) \rightarrow \mathbf{Z}_{8}^{2 g} \oplus 2 \mathbf{Z}_{8}^{(2 g}\right) \oplus 4 \mathbf{Z}_{8}^{(2 g}\right)
$$

is isomorphic.

Remark 4.10. In particular, we have Ker $\iota=\langle 1\rangle$ when $r=1$.

Now, we prove Theorem 1.2

proof of Theorem 1.2. We compute the kernel of the homomorphism $\Phi: \mathbf{Z}_{8}\left[H_{1}\left(\Sigma_{g, 1} ; \mathbf{Z}_{2}\right)\right] \rightarrow H_{1}\left(\mathcal{M}_{g, 1}[2] ; \mathbf{Z}\right)$.

Since $\beta_{\sigma}$ is injective, $\operatorname{Ker} \beta_{\sigma} \Phi=\operatorname{Ker} \Phi$. Hence, by Lemma 4.7 we have

$$
4 \Delta_{\sigma}^{2}\left(x_{1}, x_{2}\right), 2 \Delta_{\sigma}^{3}\left(x_{1}, x_{2}, x_{3}\right), \Delta_{\sigma}^{n}\left(x_{1}, x_{2}, \cdots, x_{n}\right) \in \operatorname{Ker} \Phi,
$$

for $n \geq 3$ and $\left\{x_{i}\right\}_{i=1}^{n} \subset H_{1}\left(\Sigma_{g, 1} ; \mathbf{Z}\right)$. By Lemma 4.6, it is easy to see that

$$
4 \Delta_{\sigma}^{2}\left(x_{1}, x_{2}\right), 2 \Delta_{\sigma}^{3}\left(x_{1}, x_{2}, x_{3}\right), \Delta_{\sigma}^{n}\left(x_{1}, x_{2}, \cdots, x_{n}\right)
$$

is generated by

$$
4 \Delta_{\sigma}^{2}\left(X_{i_{1}}, X_{i_{2}}\right), 2 \Delta_{\sigma}^{3}\left(X_{i_{1}}, X_{i_{2}}, X_{i_{3}}\right), \Delta_{\sigma}^{n}\left(X_{i_{1}}, X_{i_{2}}, \cdots, X_{i_{n}}\right),
$$

where $\left\{X_{i}\right\}_{i=1}^{2 g} \subset H_{1}\left(\Sigma_{g, 1} ; \mathbf{Z}\right)$ is the symplectic basis. Hence, the submodule $L_{g, 1}$ is generated by these elements. An easy calculation shows that

$$
\left|\mathbf{Z}_{8}\left[H_{1}\left(\Sigma_{g, 1} ; \mathbf{Z}_{2}\right)\right] / L_{g, 1}\right|=\left|\mathbf{Z}_{8}^{2 g} \oplus \mathbf{Z}_{4}^{(2 g)} \oplus \mathbf{Z}_{2}^{\left(\begin{array}{c}
2 g \\
3
\end{array}\right)}\right|=\left|H_{1}\left(\mathcal{M}_{g, 1}[2] ; \mathbf{Z}\right)\right| .
$$


Therefore, the surjective homomorphism

$$
\Phi: \mathbf{Z}_{8}\left[H_{1}\left(\Sigma_{g, 1} ; \mathbf{Z}_{2}\right)\right] / L_{g, 1} \rightarrow H_{1}\left(\mathcal{M}_{g, 1}[2] ; \mathbf{Z}\right)
$$

is isomorphic.

If we choose the spin structure $\sigma_{0}$ such that its quadratic function $q_{\sigma_{0}}$ satisfies $q_{\sigma_{0}}\left(X_{i}\right)=0$, we have

$$
q_{\sigma_{0}}\left(x_{1}+x_{2}+\cdots+x_{n}\right)=\sum_{1 \leq i<j \leq n}\left(x_{i} \cdot x_{j}\right) \bmod 2=I\left(x_{1}, x_{2}, \cdots, x_{n}\right) .
$$

Hence we have $\Delta_{0}=\Delta_{\sigma_{0}}$, and Theorem 1.2 is proved.

\subsection{The abelianization of the level 2 mapping class group of a closed surface}

In this subsection, we determine the abelianization of the level 2 mapping class group of a closed surface $\Sigma_{g}$. It is well-known that the homomorphism

$$
\mathcal{M}_{g, 1}[2] \rightarrow \mathcal{M}_{g}[2]
$$

is surjective. As stated in Johnson [13] Section 6, the kernel $\operatorname{Ker}\left(H_{1}\left(\mathcal{I}_{g, 1} ; \mathbf{Z}\right)_{\mathcal{M}_{g, 1}[2]} \rightarrow H_{1}\left(\mathcal{I}_{g} ; \mathbf{Z}\right)_{\mathcal{M}_{g}[2]}\right)$ is generated by

$$
\sum_{i=1}^{g} \bar{A}_{i} \bar{B}_{i}, \sum_{i=1}^{g} \bar{A}_{i} \bar{B}_{i} \bar{X} \in B_{g, 1}^{3} \quad \text { for } X=A_{1}, B_{1}, \cdots, A_{g}, B_{g} .
$$

Hence, $\operatorname{Ker}\left(H_{1}\left(\mathcal{M}_{g, 1}[2] ; \mathbf{Z}\right) \rightarrow H_{1}\left(\mathcal{M}_{g}[2] ; \mathbf{Z}\right)\right)$ is generated by the image of these elements under $\iota$. Therefore, $H_{1}\left(\mathcal{M}_{g}[2] ; \mathbf{Z}\right)$ is isomorphic to the quotient of $\mathbf{Z}_{8}\left[H_{1}\left(\Sigma_{g, 1} ; \mathbf{Z}_{2}\right)\right] / L_{g, 1}$ by the image of these elements under $\iota$.

We write $\iota\left(\bar{A}_{i} \bar{B}_{i}\right), \iota\left(\bar{A}_{i} \bar{B}_{i} \bar{X}\right)$ as elements of $\mathbf{Z}_{8}\left[H_{1}\left(\Sigma_{g, 1} ; \mathbf{Z}_{2}\right)\right] / L_{g, 1}$. As we saw in Lemma 4.5, we have

$$
\iota\left(\bar{A}_{1} \bar{B}_{1}\right)=2 \Phi \Delta_{0}^{2}\left(A_{1}, B_{1}\right)+4\left\langle A_{1}\right\rangle+4\left\langle B_{1}\right\rangle,
$$

and

$$
\begin{aligned}
\iota\left(\bar{A}_{1} \bar{B}_{1}\left(\overline{B_{2}+1}\right)\right) & =\left\langle B_{1}\right\rangle+\left\langle A_{1}\right\rangle+\left\langle B_{1}+B_{2}\right\rangle+\left\langle A_{1}+B_{1}\right\rangle+\left\langle A_{1}+B_{1}+B_{2}\right\rangle+\left\langle A_{1}+B_{2}\right\rangle-\left\langle B_{2}\right\rangle \\
& =-\Phi \Delta_{0}^{3}\left(A_{1}, B_{1}, B_{2}\right)+2 \Phi \Delta_{0}^{2}\left(A_{1}, B_{2}\right)+2 \Phi \Delta_{0}^{2}\left(B_{1}, B_{2}\right)-4\left\langle B_{2}\right\rangle \\
& =\Phi \Delta_{0}^{3}\left(A_{1}, B_{1}, B_{2}\right)+2 \Phi \Delta_{0}^{2}\left(A_{1}, B_{2}\right)+2 \Phi \Delta_{0}^{2}\left(B_{1}, B_{2}\right)+4\left\langle B_{2}\right\rangle .
\end{aligned}
$$

Hence for $X=A_{1}, B_{1}, \cdots, A_{g}, B_{g}$, we have

$$
\begin{aligned}
\iota\left(\bar{A}_{i} \bar{B}_{i}\right) & =\Phi\left\{2 \Delta_{0}^{2}\left(A_{i}, B_{i}\right)+4\left[A_{i}\right]+4\left[B_{i}\right]\right\}, \\
\iota\left(\bar{A}_{i} \bar{B}_{i} \bar{X}\right) & =\Phi\left\{\Delta_{0}^{3}\left(A_{i}, B_{i}, X\right)+2 \Delta_{0}^{2}\left(A_{i}, X\right)+2 \Delta_{0}^{2}\left(A_{i}, B_{i}\right)+2 \Delta_{0}^{2}\left(B_{i}, X\right)+4\left[A_{i}\right]+4\left[B_{i}\right]+4[X]\right\} .
\end{aligned}
$$

Proposition 4.11. Let $g \geq 3$. Denote by $L_{g}$ the submodule of $\mathbf{Z}_{8}\left[H_{1}\left(\Sigma_{g} ; \mathbf{Z}_{2}\right)\right]$ generated by

$$
\begin{gathered}
{[0], 4 \Delta_{0}^{2}\left(x_{1}, x_{2}\right), 2 \Delta_{0}^{3}\left(x_{1}, x_{2}, x_{3}\right), \Delta^{n}\left(x_{1}, x_{2}, \cdots, x_{n}\right),} \\
\sum_{i=1}^{g}\left\{2 \Delta_{0}^{2}\left(A_{i}, B_{i}\right)+4\left[A_{i}\right]+4\left[B_{i}\right]\right\}, \\
\sum_{i=1}^{g}\left\{\Delta_{0}^{3}\left(A_{i}, B_{i}, X\right)+2 \Delta_{0}^{2}\left(A_{i}, X\right)+2 \Delta_{0}^{2}\left(B_{i}, X\right)+4[X]\right\},
\end{gathered}
$$

for $\left\{x_{i}\right\}_{i=1}^{n} \subset H_{1}\left(\Sigma_{g} ; \mathbf{Z}_{2}\right)$ and $X=A_{1}, B_{1}, \cdots, A_{g}, B_{g}$. Then, we have

$$
\mathbf{Z}_{8}\left[H_{1}\left(\Sigma_{g} ; \mathbf{Z}_{2}\right)\right] / L_{g} \cong H_{1}\left(\mathcal{M}_{g}[2] ; \mathbf{Z}\right) .
$$




\section{The abelianization of the level $d$ mapping class group for odd $d$}

In this section, we prove Theorem 1.3 . The exact sequence

$$
1 \rightarrow \mathcal{I}_{g, r} \rightarrow \mathcal{M}_{g, r}[d] \rightarrow \Gamma_{g}[d] \rightarrow 1
$$

plays an important role. By the Lyndon-Hochschild-Serre spectral sequence, we have the exact sequence

$$
H_{1}\left(\mathcal{I}_{g, r} ; \mathbf{Z}\right) \rightarrow H_{1}\left(\mathcal{M}_{g, r}[d] ; \mathbf{Z}\right) \rightarrow H_{1}\left(\Gamma_{g}[d] ; \mathbf{Z}\right) \rightarrow 0 .
$$

\subsection{Mod $d$ reduction of inclusion homomorphism}

Lemma 5.1. Let $g \geq 3$. The homomorphism $H_{1}\left(\mathcal{I}_{g, r} ; \mathbf{Z}\right) \rightarrow H_{1}\left(\mathcal{M}_{g, r}[d] ; \mathbf{Z}\right)$ factors through $H_{1}\left(\mathcal{I}_{g, r} ; \mathbf{Z}\right) \otimes \mathbf{Z}_{d}$.

Proof. For any pair of simple closed curves $C_{1}, C_{1}^{\prime}$ which bounds a subsurface of genus 1 in $\Sigma_{g, 1}$, the mapping class $t_{C_{1}} t_{C_{1}^{\prime}}^{-1}$ is in Torelli group $\mathcal{I}_{g, 1}$. Johnson 9 ] showed that $\mathcal{I}_{g, 1}$ is generated by all pairs of twists $t_{C_{1}} t_{C_{1}^{\prime}}^{-1}$, for $g \geq 3$ and such an bounding pair $C_{1}, C_{1}^{\prime}$. In particular, $\mathcal{I}_{g}$ is also generated by pairs of twists as above. Johnson (11] Lemma 11) also shows that any pair of simple closed curves $C_{2}, C_{2}^{\prime}$ which bounds a subsurface in $\Sigma_{g, r}$ satisfies $\left(t_{C_{2}} t_{C_{2}^{\prime}}^{-1}\right)^{d} \in\left[\mathcal{M}_{g, r}[d], \mathcal{I}_{g, r}\right]$.

Therefore for $\varphi \in \mathcal{I}_{g, r}$, we have $\left[\varphi^{d}\right]=0 \in H_{1}\left(\mathcal{M}_{g, r}[d] ; \mathbf{Z}\right)$ for $r=0,1$. This proves the lemma.

We have already determines the abelianization of level $d$ congruence subgroup of the symplectic group in Section 2, We will construct the splitting of

$$
H_{1}\left(\mathcal{I}_{g, r} ; \mathbf{Z}\right) \otimes \mathbf{Z}_{d} \rightarrow H_{1}\left(\mathcal{M}_{g, r}[d] ; \mathbf{Z}\right) \rightarrow H_{1}\left(\Gamma_{g}[d] ; \mathbf{Z}\right) \rightarrow 0
$$

for $r=0,1$, and prove Theorem 1.3 in the next subsection.

\subsection{Johnson homomorphism $\bmod d$}

In this subsection, we state that the mod $d$ reduction of the Johnson homomorphism can be defined on the level $d$ mapping class group.

For $n \geq 2$, we denote by $F_{n}$ the free group of rank $n$, and by $H:=F_{n} /\left[F_{n}, F_{n}\right]$ the abelianization of $F_{n}$. Let $\operatorname{Aut}\left(F_{n}\right)$ be the automorphism group of the free group $F_{n}$. Then, Aut $F_{n}$ acts on $H$. For a commutative ring $R$ with unit element, denote the tensor algebra of $H \otimes R$ by

$$
\hat{T}:=\prod_{m=0}^{\infty} H^{\otimes m} \otimes R .
$$

We denote $\hat{T}_{i}:=\prod_{m \geq i} H^{\otimes i} \otimes R$ for $i \geq 1$.

Definition 5.2. The map $\theta: F_{n} \rightarrow 1+\hat{T}_{1}$ is called R-valued Magnus expansion of $F_{n}$ if $\theta: F_{n} \rightarrow 1+\hat{T}_{1}$ is a group homomorphism, and for any $\gamma \in F_{n}, \theta$ satisfies

$$
\theta(\gamma) \equiv 1+[\gamma]\left(\bmod \hat{T}_{2}\right)
$$

In detail, see Kawazumi[14] Section 1 and Bourbaki[3] Ch.2, $\S 5$, no.4, 5. In the following, we put $R:=\mathbf{Z}_{d}$ for an odd integer $d$. We denote by $\theta_{m}: F_{n} \rightarrow H^{\otimes m} \otimes \mathbf{Z}_{d}$ the $m$-th component of $\theta$. Denote the kernel

$$
\Gamma_{2}^{d}:=\operatorname{Ker}\left(F_{n} \rightarrow H \otimes \mathbf{Z}_{d}\right),
$$


then the restriction of $\theta_{2}$ to $\Gamma_{2}^{d} \rightarrow H^{\otimes 2} \otimes \mathbf{Z}_{d}$ is a homomorphism. For $a, b \in F_{n}$, denote by $A, B \in H_{1}\left(F_{n} ; \mathbf{Z}\right)$ the homology classes. Then, we have

$$
\begin{aligned}
\theta_{2}\left(a b a^{-1} b^{-1}\right) & =A \otimes B-B \otimes A, \\
\theta_{2}\left(a^{d}\right) & =\frac{d(d-1)}{2} A \otimes A=0 .
\end{aligned}
$$

Hence we obtain

$$
\theta_{2}\left(\Gamma_{2}^{d}\right)=\Lambda^{2} H \otimes \mathbf{Z}_{d}
$$

From the above calculation, we see that $\left.\theta_{2}\right|_{\Gamma_{2}^{d}}$ is Aut $F_{n}$-equivariant. Define the level $d$ IA-automorphism group by $I A_{n}[d]:=\operatorname{Ker}\left(\right.$ Aut $\left.F_{n} \rightarrow G L\left(n ; \mathbf{Z}_{d}\right)\right)$. For $H^{*}:=\operatorname{Hom}(H, \mathbf{Z})$, define the $\bmod d$ Johnson homomorphism by

$$
\begin{aligned}
\tau_{d}: \quad I A_{n}[d] & \rightarrow \operatorname{Hom}\left(H, \Lambda^{2} H \otimes \mathbf{Z}_{d}\right) \cong H^{*} \otimes \Lambda^{2} H \otimes \mathbf{Z}_{d} . \\
\varphi & \mapsto\left([x] \rightarrow \theta_{2}\left(x^{-1} \varphi(x)\right)\right)
\end{aligned}
$$

Then, we see that $\tau_{d}$ is an $\operatorname{Aut}\left(F_{n}\right)$-equivariant homomorphism, as in Johnson [10 Lemmas $2 \mathrm{C}$ and $2 \mathrm{D}$, Kawazumi 14] section 3.

Next, we state that we can define the $\bmod d$ Johnson homomorphism on the level $d$ mapping class group. Choose symplectic generators $\left\{a_{i}, b_{i}\right\}_{i=1}^{g}$ of $\pi_{1}\left(\Sigma_{g, 1}, *\right)\left(* \in \partial \Sigma_{g, 1}\right)$ which represent the symplectic basis $\left\{A_{i}, B_{i}\right\}$. Then we have the isomorphism $\pi_{1}\left(\Sigma_{g, 1}, *\right) \cong F_{2 g}$, and $H \cong H_{1}\left(\Sigma_{g, 1} ; \mathbf{Z}\right)$. The action of $\mathcal{M}_{g, 1}[d]$ on the fundamental group of the surface induces the homomorphism $\mathcal{M}_{g, 1}[d] \rightarrow I A_{n}[d]$. Hence we have the homomorphism

$$
\tau_{d}: \mathcal{M}_{g, 1}[d] \rightarrow H^{*} \otimes \Lambda^{2} H \otimes \mathbf{Z}_{d} \cong H \otimes \Lambda^{2} H \otimes \mathbf{Z}_{d}
$$

which is independent of the choice of the generators of $\pi_{1}\left(\Sigma_{g, 1}\right)$. Note that by the Poincaré duality, we have

$$
H^{*} \otimes \Lambda^{2} H \otimes \mathbf{Z}_{d} \cong H \otimes \Lambda^{2} H \otimes \mathbf{Z}_{d}
$$

It is easy to see that the restriction of $\tau_{d}$ to $\mathcal{I}_{g, 1}$ is equal to the mod $d$ reduction of the Johnson homomorphism. Now, we calculate the image of the Johnson homomorphism on the level $d$ mapping class group.

Lemma 5.3. For $g \geq 3$,

$$
\tau_{d}\left(\mathcal{M}_{g, 1}[d]\right) \subset \Lambda^{3} H \otimes \mathbf{Z}_{d}
$$

Proof. By the Theorem [2.6. $\mathcal{M}_{g, 1}[d]$ is generated by the $d$ times Dehn twists along all non-separating curves and the Torelli group $\mathcal{I}_{g, 1}$. For the simple closed curve $C_{1}$ as shown in Figure 5 , we have

$$
\tau_{d}\left(t_{C_{1}}^{d}\right)=\frac{d(d-1)}{2} B_{1} \otimes B_{1} \otimes B_{1}=0,
$$

because $d$ is odd. Since $\left.\tau_{d}\right|_{\mathcal{I}_{g, 1}}$ is equal to the $\bmod d$ reduction of the Johnson homomorphism, we also have $\tau_{d}\left(\mathcal{I}_{g, 1}\right) \subset \Lambda^{3} H \otimes \mathbf{Z}_{d}$.

Next, We will define the Johnson homomorphism $\tau_{d}$ for closed surfaces.

Lemma 5.4. We consider $\Sigma_{g, 1}$ as a subsurface of $\Sigma_{g}$. By gluing each mapping class on $\Sigma_{g, 1}$ with identity on the disk, we have the surjective homomorphism $\mathcal{M}_{g, 1}[d] \rightarrow \mathcal{M}_{g}[d]$. Then, for $g \geq 3$, the homomorphism

$$
\tau_{d}: \mathcal{M}_{g}[d] \rightarrow \Lambda^{3} H / H \otimes \mathbf{Z}_{d}
$$

is well-defined. 
Proof. It is known that $\operatorname{Ker}\left(\mathcal{M}_{g, 1}[d] \rightarrow \mathcal{M}_{g}[d]\right)$ is generated by twisting pair $T_{C} T_{C^{\prime}}^{-1}$ and separating twist $T_{\partial \Sigma_{g, 1}}$, where $\left(C, C^{\prime}\right)$ be a pair which bounds subsurface of genus $g-1$ (see Birman[2] pp156-160). By the result of Johnson [10] Lemmas $4 \mathrm{~A}$ and $4 \mathrm{~B}$, we have $\tau_{d}\left(T_{\partial \Sigma_{g, 1}}\right)=0$, and $\tau_{d}\left(T_{C} T_{C^{\prime}}^{-1}\right) \in H \subset \Lambda^{3} H$. Since $H \subset \Lambda^{3} H$ is a $S p(2 g ; \mathbf{Z})$-invariant subspace, we see that $\tau_{d}$ of the closed surface is well-defined.

We prove Theorem 1.3 using the homomorphism defined as above.

proof of Theorem 1.3. Consider the homomorphism

$$
\begin{gathered}
\tau_{d}: \mathcal{M}_{g, 1}[d] \rightarrow \Lambda^{3} H \otimes \mathbf{Z}_{d}, \\
\tau_{d}: \mathcal{M}_{g}[d] \rightarrow \Lambda^{3} H / H \otimes \mathbf{Z}_{d},
\end{gathered}
$$

defined in Lemma 5.1 By the structure of the abelianization determined in Johnson[13] Theorems 3 and $6, \tau_{d}$ induces the isomorphism

$$
\begin{gathered}
H_{1}\left(\mathcal{I}_{g, 1} ; \mathbf{Z}\right) \otimes \mathbf{Z}_{d} \cong \Lambda^{3} H \otimes \mathbf{Z}_{d}, \\
H_{1}\left(\mathcal{I}_{g} ; \mathbf{Z}\right) \otimes \mathbf{Z}_{d} \cong \Lambda^{3} H / H \otimes \mathbf{Z}_{d}
\end{gathered}
$$

when $d$ is odd. Hence, we have the splitting of the exact sequence

$$
H_{1}\left(\mathcal{I}_{g, r} ; \mathbf{Z}\right) \otimes \mathbf{Z}_{d} \rightarrow H_{1}\left(\mathcal{M}_{g, r}[d] ; \mathbf{Z}\right) \rightarrow H_{1}\left(\Gamma_{g}[d] ; \mathbf{Z}\right) \rightarrow 0(r=0,1),
$$

by the homomorphism $\tau_{d}$. This shows that

$$
H_{1}\left(\mathcal{M}_{g, r}[d] ; \mathbf{Z}\right)=\left\{\begin{array}{l}
\Lambda^{3} H \oplus H_{1}\left(\Gamma_{g}[d] ; \mathbf{Z}\right), \text { when } r=1 \\
\Lambda^{3} H / H \oplus H_{1}\left(\Gamma_{g}[d] ; \mathbf{Z}\right), \text { when } r=0
\end{array}\right.
$$

This proves the theorem.

\section{References}

[1] H. Bass, J. Milnor, and J.P. Serre, Solution of the congruence subgroup problem for $\mathrm{SL}_{n}(n \geq 3)$ and $\mathrm{Sp}_{2 n}$ ( $n \geq 2$ ), Publications Mathématiques de L'IHÉS 33 (1967), no. 1, 59-137.

[2] J.S. Birman, Braids, Links, and Mapping Class Groups.(AM-82), Princeton University Press, 1975.

[3] N. Bourbaki, Groupes et algèbres de Lie, Hermann, 1972.

[4] B. Farb, Some Problems on Mapping Class Groups and Moduli Space, Problems on Mapping Class Groups And Related Topics 74 (2006).

[5] R. Hain, Torelli groups and Geometry of Moduli Spaces of Curves, Current Topics in Complex Algebraic Geometry (CH Clemens and J. Kollar, eds.) MSRI publications 28 (1995), 97-143.

[6] A. Heap, Bordism Invariants of the Mapping Class Group, Arxiv preprint math.GT/0502587 (2005).

[7] S.P. Humphries, Normal closures of powers of Dehn twists in mapping class groups, Glasgow J. Math 34 (1992), 313-317.

[8] J. Igusa, On the Graded Ring of Theta-Constants, American Journal of Mathematics 86 (1964), no. 1, 219-246. 
[9] D. Johnson, Homeomorphisms of a Surface which Act Trivially on Homology, Proceedings of the American Mathematical Society 75 (1979), no. 1, 119-125.

[10] _ An abelian quotient of the mapping class group $\mathcal{I}_{g}$, Mathematische Annalen 249 (1980), no. 3, $225-242$.

[11] _ Conjugacy relations in subgroups of the mapping class group and a group-theoretic description of the Rochlin invariant, Mathematische Annalen 249 (1980), no. 3, 243-263.

[12] _ Quadratic Forms and the Birman-Craggs Homomorphisms, Transactions of the American Mathematical Society 261 (1980), no. 1, 235-254.

[13] _ The structure of the Torelli Group III: The abelianization of $\mathcal{I}_{g}$, Topology 24 (1985), no. 2, 127144.

[14] N. Kawazumi, Cohomological Aspects of Magnus Expansions, Arxiv preprint math.GT/0505497 (2005).

[15] R.C. Kirby and L.R. Taylor, Pin structures on low-dimensional manifolds, Geometry of low-dimensional manifolds 2, 177-242.

[16] R. Lee, E. Miller, and S. Weintraub, The Rochlin invariant, theta functions and the holonomy of some determinant line bundle, J. reine angew. Math 392 (1988), 187-218.

[17] R. Lee and R.H. Szczarba, On the homology and cohomology of congruence subgroups, Inventiones Mathematicae 33 (1976), no. 1, 15-53.

[18] J.D. McCarthy, On the first cohomology group of cofinite subgroups in surface mapping class groups, Topology 40 (2000), no. 2, 401-418.

[19] J. Mennicke, Zur Theorie der Siegelschen Modulgruppe, Mathematische Annalen 159 (1965), no. 2, 115-129.

[20] A. Putman, The abelianization of the level L mapping class group, arXiv:math.GT/0803.0539 (2007).

[21] M. Sato, On symmetric mapping class groups, Master's thesis, University of Tokyo, (2007) (Japanese).

[22] T. Satoh, The abelianization of the congruence IA-automorphism group of a free group, Mathematical Proceedings of the Cambridge Philosophical Society 142 (2007), no. 02, 239-248.

Corrigendum ibid. 143 (2007), no. 1 255-256.

[23] V.G. Turaev, Cohomology rings, linking forms and invariants of spin structures of three-dimensional manifolds, Math. USSR Sbornik 48 (1984), no. 1, 65-79.

Masatoshi Sato

Graduate School of Mathematical Sciences,

The University of Tokyo,

3-8-1 Komaba Meguro-ku Tokyo 153-0041, Japan

E-mail: sato@ms.u-tokyo.ac.jp 